\title{
Forage Growth and Productivity of Pearl Millet as Affected by Soil Mulching, Planting Date under Salinity Conditions
}

\author{
M.Sh. Reiad, Maha M.A. Hamada, M.Sh. Abd EL- \\ Maaboud" and M.H.Khalil" \\ Agronomy Dept. Fac. Agric., Ain Shams Univ. and *Plant \\ Production Dept., Desert Research Center, Cairo, Egypt.
}

\begin{abstract}
7 FIELD experiments were carried out, at Ras Sudr Experimental Station, South Sinai Governorate, Egypt, during the two summer growing seasons 2011 and 2012. This work was conducted to study the effect of three sowing dates, i.e. $1^{\text {st }}$ May, $15^{\text {th }}$ May and $1^{\text {st }}$ June and four soil surface mulching treatments (i.e. without, plastic sheet, one and two tons rice straw/fed) under two levels of saline water irrigation conditions, i.e. 4500 and $8000 \mathrm{ppm}$ on some growth characters and forage yield of pearl millet [Pennisetum glaucum (L.) R. Br]. Obtained results showed that the growth characters and forage yield of pearl millet, i.e. plant height, number of tillers/plant, number of leaves/plant, leaf area index, leaves dry weight, stem dry weight and total plant dry weight reduced significantly by increasing saline water irrigation levels from 4500 to $8000 \mathrm{ppm}$. Early sowing date, at $1^{\text {st }}$ May, produced the highest significant values of the most growth characters and the total forage dry weight. Meantime, the growth traits and forage dry yield were significantly affected by using 2 ton of rice straw/fed and plastic sheet soil mulching treatments which produced the highest values of leaves, stem and total dry weights/fed, compared to unmulching or using one ton rice straw/fed treatments.
\end{abstract}

Keywords: Pearl millet [Pennisetum glaucum (L.) R. Br] , Saline water irrigation, Sowing date, Soil surface mulching, Rice straw, Plastic sheet, Dry forage yield.

Pearl millet [Pennisetum glaucum (L.) R. Br] is one of the major cereal crops of the semi-arid regions of Africa and Asia and it is certainly the mainstay for millions of people in the Sahel. It's grown as grain and fodder crop (Blummel et al., 2003). In Egypt, pearl millet is not the staple food of rural populations as in the other countries of Africa, but using as summer fodder crop. South Sinai, as arid region with low rainfall and high evapo-transpiration (ET), brackish or saline groundwater is the main source of water for both domestic and agriculture use. The fodder demand is mostly faced by the local production of alfalfa and some annual forage cereals, as barley and sorghum, for feeding goats, sheep, cattle or camel. However, pearl millet is rated to be fairly tolerant to salinity (Krishnamurthy et al., 2007) and superiority over wheat, corn, and sorghum under dry and saline conditions in semi-arid land (Ferraris, 1973). Yakubu et al. 
(2010) reported that, plant height, shoot and root dry weights of five pearl millet varieties were significantly decreased with increasing soil salinization. Also, Leila Radhouane (2013) used three levels of saline water irrigation, $\mathrm{T}_{1}(1 \mathrm{~g}$ $\mathrm{NaCl} / 1$ as control), $\mathrm{T}_{2}(4 \mathrm{~g} \mathrm{NaCl} / \mathrm{l})$ and $\mathrm{T}_{3}(7 \mathrm{~g} \mathrm{NaCl} / \mathrm{l})$ for pearl millet plants, noticed that plant height of $\mathrm{T}_{3}$ was $18 \%$ significantly lower than $\mathrm{T}_{1}$ and $13 \%$ than $\mathrm{T}_{2}$. The same findings were noticed by Abd El-Rahman et al. (2005), Hussein et al. (2010) and Siti Aishah et al. (2011) on sorghum.

Concerning sowing dates, Hancock \& Greg Durham (2010), used five planting dates for pearl millet [Pennisetum glaucum (L.) R. Br.], found that the total DM yields were the highest in the late April planting and decreased linearly (by as much as $80 \mathrm{lb} / \mathrm{acre}$ ) for each day plantings were delayed past late April in 2008 and 2009 and quadratically during the extreme drought conditions of 2007. They added that late planting date influences the yield of pearl millet forage, staggered plantings are recommended to better distribute forage production throughout the growing season. Also, Maas et al. (2007) found that there was a significant decrease in grain yield, as well as plant height by delaying sowing dates. However, Wailare (2009) observed that sowing dates $\left(2^{\text {nd }}\right.$ week of July, $3^{\text {rd }}$ week of July, and $4^{\text {th }}$ week of July spaced at one week interval at Bagauda, Kano State, Nigeria) did not have significant influence on plant height of pearl millet.

Regarding soil mulching, Wang et al. (2001) found that covering the soil surface with plant residues can reduce soil evaporation. They added that wheat straw mulch reduced evaporation by $50 \%$ under winter wheat and this is equivalent to about $80 \mathrm{~mm}$ of water in the north China plain. Uwahm \& Iwo (2011), used organic mulch rates $(0,2,4,6$, and $8 \mathrm{t} / \mathrm{ha})$ on maize productivity (Zea mays L.), found that soil moisture reserves, plant height and number of leaves/plant were highest at the 8 t/ha mulch rate, followed by 6 t/ha rate. Whereas, the unmulching as control plots had the lowest soil moisture reserves, shortest plants and least number of leaves/plant. Kobayashi et al. (2010) investigated how to apply pearl millet stalk for improving soil moisture condition for plant growth of pearl millet, using 3 irrigation intensities (the $1^{\text {st }}$ was irrigated at 3-6 $\mathrm{mm} /$ day as standard, the $2^{\text {nd }}$ was at $2 / 3$ of standard as slightly watersaving, and the $3^{\text {rd }}$ was at $1 / 3$ of standard as water-saving) and 3 treatments of stalk application (stubble mulching with pearl millet stalk residues, mixing soil with pearl millet stalk residues and non-treatment as control). They indicated that plant growth of pearl millet, above ground surface, did not show significant differences among the plants under the different irrigation intensities. Under nontreatment condition, pearl millet even in the $2 / 3$ and $1 / 3$ water-saving grew as well as that in the standard treatment. However, the fresh weights of non-treated was significantly lower than that of the other treatments, stubble mulching or mixing soil with pearl millet stalk residues. Additionally, there was a tendency for pearl millet growth of stubble mulching to higher than that of mixing soil with pearl millet stalk residues. This implies that mulching with pearl millet stalk residues can be more effective on plant growth. 


\section{Material and Methods}

Two field experiments were conducted, at Ras-Sudr Experimental Station, South of Sinia Governorate, in two successive summer seasons, 2011 and 2012, to study the effect of saline water irrigation, sowing date and soil mulching on growth and forage yield of pearl millet (Pennisetum glaucum (L.) R.Br.) cv. Shandaweel 1. Each experiment included twenty four treatments, which were the combination between two levels, i.e. 4500 and $8000 \mathrm{ppm}$ of saline water irrigation, three sowing dates, i.e. $1^{\text {st }}$ May, $15^{\text {th }}$ May and $1^{\text {st }}$ June and four soil mulching treatments, i.e. without mulching, plastic sheet, 1 and 2 tons rice straw per fed. Each experiment was laid in a split-split plot design with three replicates. Saline water irrigation levels were arranged in the main plots, sowing dates were allocated in the sub plots, and soil mulching treatments were assigned in the sub-sub plots. The plot area was $10.5 \mathrm{~m}^{2}(3 \mathrm{~m} \times 3.5 \mathrm{~m})$ and contained four furrows $(3.5 \mathrm{~m}$ in length and $60 \mathrm{~cm}$ apart). Pearl millet seeds were sown, at the above mentioned sowing dates, in hills (about 5 seeds/hill, $20 \mathrm{~cm}$ apart) on the two ridges of furrows. Prior to planting, organic manure and calcium super phosphate $\left(15.5 \% \mathrm{P}_{2} \mathrm{O}_{5}\right)$ were added at the rate of $20 \mathrm{~m}^{3}$ and $200 \mathrm{~kg}$ per fed, respectively during the soil preparation. Nitrogen fertilizers were added at $60 \mathrm{~kg} / \mathrm{fed}$, as ammonium sulphate $(20.5 \%)$ in three equal doses, the $1^{\text {st }}$ dose was applied after 3 weeks from sowing dates, the $2^{\text {nd }}$ and $3^{\text {rd }}$ doses were applied after two weeks from the later cuts just before irrigation. The irrigation water was added as immersion in the two seasons. The lowest saline water irrigation level $(4500 \mathrm{ppm})$ produced three cuts at the $1^{\text {st }}$ and the $2^{\text {nd }}$ sowing dates, and two cuts at the $3^{r d}$ sowing date. On the other hand, the highest saline water irrigation level $(8000 \mathrm{ppm})$ produced two cuts at the $1^{\text {st }}$ and the $2^{\text {nd }}$ sowing dates. Whereas, produced one cut at the $3^{\text {rd }}$ sowing date. The $1^{\text {st }}$ cut was taken after 60 days from sowing date, the $2^{\text {nd }}$ cut was taken after 45 days from the $1^{\text {st }}$ cut date and the $3^{\text {rd }}$ cut was taken after 45 days from the $2^{\text {nd }}$ cut date.

Physical and chemical analysis of the experimental field soil was determined as shown in Table 1. Mechanical analysis was carried out according to Jackson (1958). Chemical analysis was carried out according to Jackson (1958) and Chapman \& Pratt (1961). The soil texture of this site was sandy loam containing calcium carbonate of 58.99 and $61.28 \%$ in the $1^{\text {st }}$ and $2^{\text {nd }}$ seasons, respectively. The mechanical, physical and chemical analysis of the experimental soil is shown in Table 1. The chemical analysis of saline water irrigation is shown in Table 2.

Data were subjected to the proper statistical analysis of variance and the combined analysis for the results of the two seasons was applied according to Steel \& Torrie (1960). The treatment means were compared as showed by Waller \& Duncan (1969). 
TABLE 1. Mechanical, physical and chemical analysis of the experiment soil. a: Mechanical and physical analysis.

\begin{tabular}{|c|c|c|c|c|c|c|}
\hline $\begin{array}{c}\text { Depth } \\
\text { (cm) }\end{array}$ & $\begin{array}{c}\text { Coarse } \\
\text { sand } \\
(0.5-1.0 \\
\text { mm }) \\
\end{array}$ & $\begin{array}{c}\text { Fine sand } \\
(0.1-0.25 \\
\text { mm })\end{array}$ & $\begin{array}{c}\text { Silt } \\
(0.002-0.05 \\
\text { mm })\end{array}$ & $\begin{array}{c}\text { Total } \\
\text { sand }(0.1- \\
1 \text { mm })\end{array}$ & $\begin{array}{c}\text { Clay } \\
(<0.002 \\
\text { mm })\end{array}$ & $\begin{array}{l}\text { Class } \\
\text { texture }\end{array}$ \\
\hline \multicolumn{7}{|c|}{$1^{s t}$ season $(2011)$} \\
\hline $0-30$ & 21.84 & 57.73 & 9.5 & 79.57 & 10.91 & Sandy loam \\
\hline $30-60$ & 22.17 & 63.45 & 6.987 & 85.62 & 7.39 & Sandy loam \\
\hline \multicolumn{7}{|c|}{$2^{\text {nd }}$ season $(2012)$} \\
\hline $0-30$ & 20.65 & 58.63 & 11.47 & 79.28 & 9.25 & Sandy loam \\
\hline $30-60$ & 23.21 & 61.53 & 7.86 & 84.74 & 7.40 & Sandy loam \\
\hline
\end{tabular}

b: Chemical analysis

\begin{tabular}{|c|c|c|c|c|c|c|c|c|c|c|c|c|c|c|}
\hline \multirow{3}{*}{ 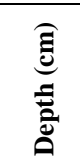 } & \multirow{3}{*}{ 플 } & \multirow{3}{*}{ 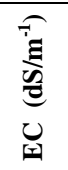 } & \multirow{3}{*}{ 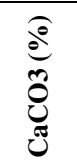 } & \multicolumn{7}{|c|}{$\begin{array}{c}\text { Saturation soluble extract } \\
(\mathrm{mg} / \mathbf{1 0 0 g})\end{array}$} & \multicolumn{4}{|c|}{$\begin{array}{c}\text { Available nutrients } \\
\left(\mathrm{mg} \mathrm{Kg} \mathrm{Kg}^{-1}\right)\end{array}$} \\
\hline & & & & \multicolumn{3}{|c|}{ Cations } & \multicolumn{4}{|c|}{ Anions } & \multirow{2}{*}{$\mathbf{N}$} & \multirow{2}{*}{$\mathbf{P}$} & \multirow{2}{*}{$\mathbf{K}$} & \multirow{2}{*}{$\mathbf{F e}$} \\
\hline & & & & $\mathrm{Ca}^{++}$ & $\mathbf{M g}^{++}$ & $\mathrm{Na}^{+}$ & $\mathrm{CO}_{3}^{-}$ & $\mathrm{HCO}_{3}=$ & $\mathrm{Cl}^{-}$ & $\mathrm{SO}_{4}{ }^{=}$ & & & & \\
\hline \multicolumn{15}{|c|}{$1^{\text {st }}$ season $(2011)$} \\
\hline $0-30$ & 7.7 & 8.60 & 58.99 & 24.5 & 5.2 & 57.1 & 0.0 & 6.0 & 61.5 & 22.2 & 26.0 & 5.1 & 51.5 & 4.2 \\
\hline $30-60$ & 7.9 & 7.35 & 52.48 & 16.7 & 3.7 & 49.5 & 0.0 & 3.5 & 49.0 & 19.5 & 18.5 & 3.3 & 35.3 & 3.4 \\
\hline \multicolumn{15}{|c|}{$2^{\text {nd }}$ season (2012) } \\
\hline $0-30$ & 7.8 & 8.82 & 61.28 & 25.1 & 5.7 & 57.8 & 0.0 & 6.2 & 61.7 & 23.4 & 26.2 & 5.2 & 51.5 & 4.3 \\
\hline $30-60$ & 7.9 & 7.50 & 55.71 & 17.3 & 4.2 & 49.8 & 0.0 & 3.7 & 50.1 & 20.3 & 18.6 & 3.6 & 35.4 & 3.6 \\
\hline
\end{tabular}

TABLE 2. Chemical analysis of the irrigation water.

\begin{tabular}{|c|c|c|c|c|c|c|c|c|c|c|}
\hline \multirow{2}{*}{ Wells } & \multirow{2}{*}{$\begin{array}{c}\text { EC } \\
(\mathbf{p p m})\end{array}$} & \multirow{2}{*}{$\mathbf{p H}$} & \multicolumn{4}{|c|}{ Soluble anions (meq/l) } & \multicolumn{4}{|c|}{ Soluble cations (meq/l) } \\
\hline & & & $\mathrm{CO3}^{=}$ & $\mathrm{HCO}_{3}^{-}$ & $\mathrm{SO}_{4}=$ & $\mathrm{Cl}^{-}$ & $\mathrm{Ca}^{++}$ & $\mathrm{Mg}^{++}$ & $\mathrm{Na}^{+}$ & $\mathbf{K}^{+}$ \\
\hline $1^{s t}$ well & 4500 & 7.5 & 0.00 & 1.68 & 23.80 & 32.16 & 14.11 & 8.30 & 32.11 & 0.30 \\
\hline $2^{\text {nd }}$ well & 8000 & 7.9 & 0.00 & 3.27 & 37.85 & 60.19 & 20.65 & 19.11 & 53.50 & 0.43 \\
\hline
\end{tabular}

\section{Results and Disscution}

Results obtained in Table 3 showed that fresh and dry weight of pearl millet yield decreased significantly by increasing irrigation water salinity from $4500 \mathrm{ppm}$ to $8000 \mathrm{ppm}$. These results could be due to the effect of high salinity of the irrigation water at $8000 \mathrm{ppm}$ which decreased all growth parameters, i.e. plant height, leaf area $/ \mathrm{m}^{2}$, leaf area index and number of tillers $/ \mathrm{m}^{2}$. In this respect, Zeinolabedin (2012) reported that, general symptoms of damage by salt stress are growth inhibition, accelerated development and senescence and death 
during prolonged exposure. He added that growth inhibition is the primary injury that leads to other symptoms although programmed cell death may also occur under severe salinity shock. Yakubu et al. (2010) reported that germination percentage, plant height, shoot and root dry weights of some millet varieties were significantly decreased with increasing soil salinization. Also, Abd El-Rahman et al. (2005) studied the effect of salinity on productivity of some Sudan grass varieties (Sorghum bicolor) var. Sudanense. They noticed that all different traits, i.e. plant height, number of tillers/plant, number of leaves/plant, leaf area, fresh and dry weights of stem + sheaths/plant, stem + sheaths/blades ratio, fresh and dry weights of forage yield/fed were significantly decreased with increasing saline water irrigation levels from 3700 to 9200 ppm. Mean time, Siti Aishah et al. (2011) found an inverse relationship between increasing salinity and dry forage yield of sorghum.

TABLE 3. Effect of saline water irrigation levels on some growth characters and forage yield of pearl millet (average the two seasons 2011 and 2012).

\begin{tabular}{|c|c|c|c|c|}
\hline \multirow{2}{*}{$\begin{array}{c}\text { Cutting } \\
\text { No. }\end{array}$} & \multicolumn{4}{|c|}{ Saline water irrigation levels (ppm) } \\
\hline & 4500 & 8000 & 4500 & 8000 \\
\hline & \multicolumn{2}{|c|}{ Plant ht. (cm) } & \multicolumn{2}{|c|}{ No. of tillers $/ \mathrm{m}^{2}$} \\
\hline $1^{\text {st }}$ cut & $\begin{array}{c}99.44 \\
\mathrm{a}\end{array}$ & $\begin{array}{c}90.84 \\
\text { b }\end{array}$ & $\begin{array}{c}119.8 \\
\mathrm{a}\end{array}$ & $\begin{array}{c}67.47 \\
b\end{array}$ \\
\hline $2^{\text {nd }}$ cut & $\begin{array}{c}118.2 \\
\mathrm{a}\end{array}$ & $\begin{array}{c}64.54 \\
b\end{array}$ & $\begin{array}{c}91.79 \\
\mathrm{a}\end{array}$ & $\begin{array}{c}27.06 \\
b\end{array}$ \\
\hline \multirow[t]{2}{*}{$3^{\text {rd }}$ cut } & $\begin{array}{c}78.84 \\
a\end{array}$ & $\begin{array}{l}0 \\
\mathrm{~b}\end{array}$ & $\begin{array}{c}19.84 \\
\mathrm{a}\end{array}$ & $\begin{array}{l}0 \\
b\end{array}$ \\
\hline & \multicolumn{2}{|c|}{ Leaves area $\left(\mathrm{cm}^{2} / \mathrm{m}^{2}\right)$} & \multicolumn{2}{|c|}{ Leaf area index } \\
\hline $1^{s t}$ cut & $\begin{array}{c}77610 \\
\mathrm{a}\end{array}$ & $\begin{array}{c}12720 \\
\text { b }\end{array}$ & $\begin{array}{c}7.761 \\
\mathrm{a}\end{array}$ & $\begin{array}{c}1.272 \\
\mathrm{~b}\end{array}$ \\
\hline $2^{\text {nd }}$ cut & $\begin{array}{c}61730 \\
a\end{array}$ & $\begin{array}{c}4127 \\
b\end{array}$ & $\begin{array}{c}6.173 \\
\mathrm{a}\end{array}$ & $\begin{array}{c}0.413 \\
\mathrm{~b}\end{array}$ \\
\hline $3^{\text {rd }}$ cut & $\begin{array}{c}8098 \\
\text { a }\end{array}$ & $\begin{array}{l}0 \\
b\end{array}$ & $\begin{array}{c}0.810 \\
\mathrm{a}\end{array}$ & $\begin{array}{l}0 \\
b\end{array}$ \\
\hline & \multicolumn{2}{|c|}{ Leaves dry wt. (t/fed) } & \multicolumn{2}{|c|}{ Stem dry wt. (t/fed.) } \\
\hline $1^{s t}$ cut & $\begin{array}{c}0.474 \\
\mathrm{a}\end{array}$ & $\begin{array}{c}0.260 \\
\mathrm{~b}\end{array}$ & $\begin{array}{c}0.313 \\
\mathrm{a} \\
\end{array}$ & $\begin{array}{c}0.228 \\
\mathrm{~b}\end{array}$ \\
\hline $2^{\text {nd }}$ cut & $\begin{array}{c}0.406 \\
a\end{array}$ & $\begin{array}{c}0.138 \\
b\end{array}$ & $\begin{array}{c}0.354 \\
\mathrm{a}\end{array}$ & $\begin{array}{c}0.159 \\
b\end{array}$ \\
\hline $3^{\text {rd }}$ cut & $\begin{array}{c}0.107 \\
\mathrm{a}\end{array}$ & $\begin{array}{l}0 \\
\mathrm{~b}\end{array}$ & $\begin{array}{c}0.113 \\
\mathrm{a}\end{array}$ & $\begin{array}{l}0 \\
\mathrm{~b}\end{array}$ \\
\hline & \multicolumn{2}{|c|}{ Total plant dry wt.(t/fed) } & \multicolumn{2}{|c|}{ Leaf/stem ratio } \\
\hline $1^{s t}$ cut & $\begin{array}{c}0.787 \\
\mathrm{a} \\
\end{array}$ & $\begin{array}{c}0.489 \\
\mathrm{~b}\end{array}$ & $\begin{array}{c}1.558 \\
\mathrm{a}\end{array}$ & $\begin{array}{c}1.156 \\
\mathrm{~b}\end{array}$ \\
\hline $2^{\text {nd }}$ cut & $\begin{array}{c}0.760 \\
\mathrm{a} \\
\end{array}$ & $\begin{array}{c}0.298 \\
\mathrm{~b}\end{array}$ & $\begin{array}{c}1.152 \\
\mathrm{a}\end{array}$ & $\begin{array}{c}0.595 \\
\mathrm{~b}\end{array}$ \\
\hline $3^{\text {rd }}$ cut & $\begin{array}{c}0.222 \\
\mathrm{a}\end{array}$ & $\begin{array}{l}0 \\
\mathrm{~b}\end{array}$ & $\begin{array}{c}0.631 \\
\mathrm{a}\end{array}$ & $\begin{array}{l}0 \\
\mathrm{~b}\end{array}$ \\
\hline
\end{tabular}

ppm $=$ part per milion, No.= number, ht. $=$ height, $w t .=$ weight, $\mathrm{t}=$ ton, fed $=$ feddan. 
Results in Table 4 indicated that sowing date had significantly effect in some growth characters and the dry weight of pearl millet under salinity conditions. Where, early planting date at $1^{s t}$ May produced a significant values of plant height, number of tillers, leaves dry weight, stem dry weight and the total dray weight per feddan in the $2^{\text {nd }}$ and $3^{\text {rd }}$ cuts. Whereas, sowing date at $1^{\text {st }}$ June produced the high significant values of plant height and leaves dry weight at the $1^{s t}$ cut. These results were inharmony with Wailare (2009), who observed that sowing dates did not have significant influence on plant height, panicle weight, number of panicles per plot, panicle length, panicle diameter, and weight of 1000 grains but stover yield and grain yield per hectare were both significantly influenced by sowing date.

TABLE 4. Effect of sowing date on some growth characters and forage yield of pearl millet (average the two seasons 2011 and 2012).

\begin{tabular}{|c|c|c|c|c|c|c|}
\hline & \multicolumn{6}{|c|}{ Sowing date } \\
\hline \multirow[t]{2}{*}{ Cutting No. } & $1 / 5$ & $15 / 5$ & $1 / 6$ & $1 / 5$ & $15 / 5$ & $1 / 6$ \\
\hline & \multicolumn{3}{|c|}{ Plant ht. (cm) } & \multicolumn{3}{|c|}{ No. of tillers $/ \mathrm{m}^{2}$} \\
\hline $1^{\text {st }} \mathrm{cut}$ & $\begin{array}{c}92.18 \\
b\end{array}$ & $\begin{array}{c}95.49 \\
\mathrm{a}\end{array}$ & $\begin{array}{c}97.75 \\
\mathrm{a}\end{array}$ & $\begin{array}{c}105.2 \\
\mathrm{a}\end{array}$ & $\begin{array}{c}88.20 \\
\text { b }\end{array}$ & $\begin{array}{c}87.39 \\
\text { b }\end{array}$ \\
\hline $2^{\text {nd }}$ cut & $\begin{array}{c}110.6 \\
\mathrm{a}\end{array}$ & $\begin{array}{c}106.3 \\
b\end{array}$ & $\begin{array}{c}57.14 \\
\mathrm{c}\end{array}$ & $\begin{array}{c}70.83 \\
\mathrm{a}\end{array}$ & $\begin{array}{c}69.97 \\
\mathrm{a}\end{array}$ & $\begin{array}{c}37.48 \\
\text { b }\end{array}$ \\
\hline $3^{\text {rd }}$ cut & $\begin{array}{c}61.21 \\
\mathrm{a}\end{array}$ & $\begin{array}{c}57.05 \\
\mathrm{~b}\end{array}$ & $\begin{array}{l}0 \\
\mathrm{c}\end{array}$ & $\begin{array}{c}16.38 \\
\mathrm{a}\end{array}$ & $\begin{array}{c}13.39 \\
\mathrm{~b}\end{array}$ & $\begin{array}{l}0 \\
\mathrm{c}\end{array}$ \\
\hline & \multicolumn{3}{|c|}{ Leaves area $\left(\mathrm{cm}^{2} / \mathrm{m}^{2}\right)$} & \multicolumn{3}{|c|}{ Leaf area index } \\
\hline $1^{\text {st }}$ cut & $\begin{array}{c}47220 \\
\mathrm{a}\end{array}$ & $\begin{array}{c}39030 \\
\text { b }\end{array}$ & $\begin{array}{c}49250 \\
\text { a }\end{array}$ & $\begin{array}{c}4.722 \\
\mathrm{a}\end{array}$ & $\begin{array}{c}3.993 \\
\text { b }\end{array}$ & $\begin{array}{c}4.925 \\
\mathrm{a}\end{array}$ \\
\hline $2^{\text {nd }}$ cut & $\begin{array}{c}50910 \\
\mathrm{a}\end{array}$ & $\begin{array}{c}36610 \\
\text { b }\end{array}$ & $\begin{array}{c}11270 \\
\mathrm{c}\end{array}$ & $\begin{array}{c}5.091 \\
\mathrm{a}\end{array}$ & $\begin{array}{c}3.661 \\
b\end{array}$ & $\begin{array}{c}1.127 \\
\mathrm{c}\end{array}$ \\
\hline $3^{\text {rd }}$ cut & $\begin{array}{c}6332 \\
\mathrm{a} \\
\end{array}$ & $\begin{array}{c}5815 \\
\mathrm{a} \\
\end{array}$ & $\begin{array}{l}0 \\
\mathrm{~d}\end{array}$ & $\begin{array}{c}0.633 \\
\mathrm{a} \\
\end{array}$ & $\begin{array}{c}0.582 \\
\mathrm{a} \\
\end{array}$ & $\begin{array}{l}0 \\
\mathrm{~b}\end{array}$ \\
\hline & \multicolumn{3}{|c|}{ Leaves dry wt. (t/fed) } & \multicolumn{3}{|c|}{ Stem dry wt. (t/fed) } \\
\hline $1^{\text {st }}$ cut & $\begin{array}{c}0.358 \\
\mathrm{~b}\end{array}$ & $\begin{array}{c}0.388 \\
\mathrm{a}\end{array}$ & $\begin{array}{c}0.356 \\
\mathrm{~b}\end{array}$ & $\begin{array}{c}0.243 \\
\mathrm{~b}\end{array}$ & $\begin{array}{c}0.263 \\
\mathrm{~b}\end{array}$ & $\begin{array}{c}0.305 \\
\mathrm{a}\end{array}$ \\
\hline $2^{\text {nd }}$ cut & $\begin{array}{c}0.334 \\
\mathrm{a}\end{array}$ & $\begin{array}{c}0.333 \\
\mathrm{a}\end{array}$ & $\begin{array}{c}0.150 \\
b\end{array}$ & $\begin{array}{c}0.312 \\
\mathrm{a}\end{array}$ & $\begin{array}{c}0.301 \\
\mathrm{a}\end{array}$ & $\begin{array}{c}0.156 \\
\mathrm{~b}\end{array}$ \\
\hline $3^{\text {rd }}$ cut & $\begin{array}{c}0.097 \\
\mathrm{a}\end{array}$ & $\begin{array}{c}0.063 \\
b\end{array}$ & $\begin{array}{l}0 \\
\mathrm{c}\end{array}$ & $\begin{array}{c}0.103 \\
\mathrm{a}\end{array}$ & $\begin{array}{c}0.067 \\
\text { b }\end{array}$ & $\begin{array}{l}0 \\
\mathrm{c}\end{array}$ \\
\hline & \multicolumn{3}{|c|}{ Total plant dry wt.(t/fed) } & \multicolumn{3}{|c|}{ Leaf/stem ratio } \\
\hline $1^{\text {st }} \mathrm{cut}$ & $\begin{array}{c}0.602 \\
b\end{array}$ & $\begin{array}{c}0.652 \\
a b\end{array}$ & $\begin{array}{c}0.661 \\
\mathrm{a}\end{array}$ & $\begin{array}{c}1.451 \\
\mathrm{a}\end{array}$ & $\begin{array}{c}1.466 \\
\mathrm{a}\end{array}$ & $\begin{array}{c}1.153 \\
\mathrm{~b}\end{array}$ \\
\hline $2^{\text {nd }}$ cut & $\begin{array}{c}0.646 \\
\mathrm{a}\end{array}$ & $\begin{array}{c}0.636 \\
\mathrm{a}\end{array}$ & $\begin{array}{c}0.305 \\
b\end{array}$ & $\begin{array}{c}1.040 \\
\mathrm{a}\end{array}$ & $\begin{array}{c}1.094 \\
\mathrm{a}\end{array}$ & $\begin{array}{c}0.487 \\
\text { b }\end{array}$ \\
\hline $3^{\text {rd }}$ cut & $\begin{array}{c}0.204 \\
\mathrm{a}\end{array}$ & $\begin{array}{c}0.130 \\
b\end{array}$ & $\begin{array}{l}0 \\
\mathrm{c}\end{array}$ & $\begin{array}{c}0.477 \\
\mathrm{a}\end{array}$ & $\begin{array}{c}0.469 \\
\mathrm{a}\end{array}$ & $\begin{array}{l}0 \\
\mathrm{~b}\end{array}$ \\
\hline
\end{tabular}

Egypt. J. Agron. 36, No. 1 (2014) 
Soler et al. (2008) found that the optimum planting date of some pearl millet varieties to obtain the maximum yield was between 13 and 23 May for variety Heini Kirei, while for the other varieties the planting dates were between 23 May and 2 June. Hancock \& Greg Durham (2010) reported that the total DM yields of pearl millet were the highest in the late April planting and decreased linearly (by as much as $80 \mathrm{lb} / \mathrm{acre}$ ) for each day plantings were delayed past late April in 2008 and 2009 and quadratically during the extreme drought conditions. Radhouane (2008) reported that the optimal planting time of pearl millet under Mediterranean environment conditions was between first May and early June. Whereas, he found that plant height was reduced in early and late planting dates. This may be due to changes in photoperiod which accelerated development towards reproductive stages and hence less time was available to vegetative growth. Low temperature during April and first weeks of May produced minimum plant height for early planting.

Data presented in Table 5 showed that there was significant difference among soil mulching treatments on the growth characters and the dry weight of pearl millet yield. Results indicated that soil mulching with plastic produced the high significant effect of the dry weight of the pearl millet yield at the $1^{\text {st }}$ cut. Whereas, soil mulching with $2 \mathrm{t} / \mathrm{fed}$, of rice straw produced the significant value of the dry weight of the pearl millet yield at the $3^{\text {rd }}$ cut. Mean time using plastic for soil mulching produced the high significant value of the accumulation dry weight for the three cuts of the pearl millet yield followed by using $2 \mathrm{t} / \mathrm{fed}$, of the rice straw. These results could be due to the positive correlation between the accumulation dry weight and plant height, number of tillers $/ \mathrm{m}^{2}$ and leaf area $/ \mathrm{m}^{2}$ as shown in Table 5.

It is known that the high temperatures and the high evaporation rate, enhancing of salt accumulation on soil surface subsequent to irrigations is inevitable unless using surface protection technique and this can be achieved by using soil surface mulching from cheaply materials and from crops residues.

Results presented in Table 6 showed the effect of the interaction between saline irrigation water levels and sowing date on pearl millet growth. Data showed that the growth and the dry yield of pearl mill significantly affected by the interaction between saline water irrigation and sowing date at the three cuts. Where, the high significant values of plant height, total yield of dry weight, leaves dry weight and stem dry weight were recorded at the third sowing date $1^{\text {st }}$ June under the low saline irrigation level (4500 ppm). Whereas, the significant values of the above characters were recorded for the first sowing date $1^{\text {st }}$ May under the low saline irrigation level $(4500 \mathrm{ppm})$, On the other hand, the lowest significant values of all studied characters were obtained under the high salinity level $(8000 \mathrm{ppm})$ and there was no re-growth of the pearl millet plants in the $2^{\text {nd }}$ cut under the high salinity level at the $3^{r d}$ sowing date of $1^{\text {st }}$ June. Meantime, there was no re-growth of the pearl millet plants under the low saline irrigation water level $(4500 \mathrm{ppm})$ at the third sowing date $1^{\text {st }}$ June. These results may be due to the effect of the high temperature at the $3^{\text {rd }}$ sowing date $1^{\text {st }}$ June which caused high evaporation from the soil surface and negatively affected in the regrowth of the plants at the $3^{\text {rd }}$ cut time. 
TABLE 5. Effect of soil mulching on some growth characters and forage yield of pearl millet (average the two seasons 2011 and 2012).

\begin{tabular}{|c|c|c|c|c|c|c|c|c|}
\hline & \multicolumn{4}{|c|}{ Soil mulching } & \multicolumn{4}{|c|}{ Soil mulching } \\
\hline \multirow[t]{2}{*}{$\begin{array}{c}\text { Cutting } \\
\text { No. }\end{array}$} & $\begin{array}{c}\text { Without } \\
\text { mulch }\end{array}$ & $\begin{array}{c}\text { 1ton/fed } \\
\text { straw } \\
\text { rice }\end{array}$ & $\begin{array}{c}\text { 2ton/fed } \\
\text { straw } \\
\text { rice }\end{array}$ & $\begin{array}{c}\text { Plastic } \\
\text { mulching }\end{array}$ & $\begin{array}{l}\text { Without } \\
\text { mulch }\end{array}$ & $\begin{array}{c}\text { 1ton/fed } \\
\text { straw } \\
\text { rice }\end{array}$ & $\begin{array}{c}\text { 2ton/fed } \\
\text { straw } \\
\text { rice }\end{array}$ & $\begin{array}{c}\text { Plastic } \\
\text { mulching }\end{array}$ \\
\hline & \multicolumn{4}{|c|}{ Plant ht. (cm) } & \multicolumn{4}{|c|}{ No. of tillers $/ \mathrm{m}^{2}$} \\
\hline $1^{s t}$ cut & $\begin{array}{c}93.18 \\
b\end{array}$ & $\begin{array}{c}94.90 \\
b\end{array}$ & $\begin{array}{c}94.17 \\
b\end{array}$ & $\begin{array}{c}98.29 \\
\mathrm{a}\end{array}$ & $\begin{array}{c}96.88 \\
\mathrm{a}\end{array}$ & $\begin{array}{c}93.37 \\
a b\end{array}$ & $\begin{array}{c}89.57 \\
b\end{array}$ & $\begin{array}{c}94.62 \\
a\end{array}$ \\
\hline $2^{\text {nd }}$ cut & $\begin{array}{c}88.99 \\
b\end{array}$ & $\begin{array}{c}87.14 \\
b\end{array}$ & $\begin{array}{c}94.08 \\
\mathrm{a}\end{array}$ & $\begin{array}{c}95.18 \\
\mathrm{a}\end{array}$ & $\begin{array}{c}54.67 \\
b\end{array}$ & $\begin{array}{c}63.28 \\
\mathrm{a}\end{array}$ & $\begin{array}{c}60.82 \\
\mathrm{a}\end{array}$ & $\begin{array}{c}58.93 \\
\mathrm{ab}\end{array}$ \\
\hline $3^{\text {rd }}$ cut & $\begin{array}{c}35.98 \\
b\end{array}$ & $\begin{array}{c}37.91 \\
b\end{array}$ & $\begin{array}{c}40.78 \\
\mathrm{a}\end{array}$ & $\begin{array}{c}43.01 \\
\mathrm{a}\end{array}$ & $\begin{array}{c}10.18 \\
\mathrm{a}\end{array}$ & $\begin{array}{c}9.433 \\
a\end{array}$ & $\begin{array}{c}9.90 \\
\mathrm{a}\end{array}$ & $\begin{array}{c}10.17 \\
\mathrm{a}\end{array}$ \\
\hline & \multicolumn{4}{|c|}{ Leaves area $\left(\mathrm{cm}^{2} / \mathrm{m}^{2}\right)$} & \multicolumn{4}{|c|}{ Leaf area index } \\
\hline $1^{s t}$ cut & $\begin{array}{c}43800 \\
a b\end{array}$ & $\begin{array}{c}46190 \\
a b\end{array}$ & $\begin{array}{c}42240 \\
b\end{array}$ & $\begin{array}{c}48430 \\
\text { a }\end{array}$ & $\begin{array}{c}4.380 \\
a b\end{array}$ & $\begin{array}{c}4.619 \\
a b\end{array}$ & $\begin{array}{c}4.224 \\
b\end{array}$ & $\begin{array}{c}4.843 \\
\mathrm{a}\end{array}$ \\
\hline $2^{\text {nd }}$ cut & $\begin{array}{c}32280 \\
b\end{array}$ & $\begin{array}{c}36030 \\
a\end{array}$ & $\begin{array}{c}35290 \\
a b\end{array}$ & $\begin{array}{c}28120 \\
c\end{array}$ & $\begin{array}{c}3.228 \\
b\end{array}$ & $\begin{array}{c}3.603 \\
\mathrm{a}\end{array}$ & $\begin{array}{c}3.529 \\
\mathrm{ab}\end{array}$ & $\begin{array}{c}2.812 \\
\mathrm{c}\end{array}$ \\
\hline $3^{\text {rd }}$ cut & $\begin{array}{c}3646 \\
b\end{array}$ & $\begin{array}{c}4097 \\
a b\end{array}$ & $\begin{array}{c}4554 \\
\mathrm{a}\end{array}$ & $\begin{array}{c}3900 \\
a b\end{array}$ & $\begin{array}{c}0.3645 \\
\mathrm{~b}\end{array}$ & $\begin{array}{c}0.4097 \\
\mathrm{ab}\end{array}$ & $\begin{array}{c}0.4554 \\
\mathrm{a}\end{array}$ & $\begin{array}{c}0.3900 \\
a b\end{array}$ \\
\hline & \multicolumn{4}{|c|}{ Leaves dry wt. (t/fed) } & \multicolumn{4}{|c|}{ Stem dry wt. (t/fed) } \\
\hline $1^{s t} \mathrm{cut}$ & $\begin{array}{c}0.366 \\
b\end{array}$ & $\begin{array}{c}0.352 \\
b\end{array}$ & $\begin{array}{c}0.340 \\
b\end{array}$ & $\begin{array}{c}0.411 \\
\mathrm{a}\end{array}$ & $\begin{array}{c}0.271 \\
b\end{array}$ & $\begin{array}{c}0.259 \\
b\end{array}$ & $\begin{array}{c}0.239 \\
b\end{array}$ & $\begin{array}{c}0.314 \\
\mathrm{a}\end{array}$ \\
\hline $2^{\text {nd }}$ cut & $\begin{array}{c}0.227 \\
\mathrm{c}\end{array}$ & $\begin{array}{c}0.276 \\
b\end{array}$ & $\begin{array}{c}0.303 \\
\mathrm{a}\end{array}$ & $\begin{array}{c}0.283 \\
a b\end{array}$ & $\begin{array}{c}0.216 \\
\mathrm{c}\end{array}$ & $\begin{array}{c}0.249 \\
b\end{array}$ & $\begin{array}{c}0.279 \\
a b\end{array}$ & $\begin{array}{c}0.282 \\
\mathrm{a}\end{array}$ \\
\hline $3^{\text {rd }}$ cut & $\begin{array}{c}0.047 \\
\mathrm{c}\end{array}$ & $\begin{array}{c}0.056 \\
b\end{array}$ & $\begin{array}{c}0.065 \\
\mathrm{a}\end{array}$ & $\begin{array}{c}0.045 \\
\mathrm{c}\end{array}$ & $\begin{array}{c}0.048 \\
\mathrm{c}\end{array}$ & $\begin{array}{c}0.057 \\
b\end{array}$ & $\begin{array}{c}0.070 \\
\mathrm{a}\end{array}$ & $\begin{array}{c}0.051 \\
\mathrm{bc}\end{array}$ \\
\hline & \multicolumn{4}{|c|}{ Total plant dry wt.(t/fed) } & \multicolumn{4}{|c|}{ Leaf/stem ratio } \\
\hline $1^{\mathrm{st}} \mathrm{cut}$ & $\begin{array}{c}0.637 \\
b\end{array}$ & $\begin{array}{c}0.611 \\
b\end{array}$ & $\begin{array}{c}0.579 \\
b\end{array}$ & $\begin{array}{c}0.726 \\
a\end{array}$ & $\begin{array}{c}1.351 \\
\mathrm{a}\end{array}$ & $\begin{array}{c}1.350 \\
\mathrm{a}\end{array}$ & $\begin{array}{c}1.414 \\
\mathrm{a}\end{array}$ & $\begin{array}{c}1.313 \\
\mathrm{a}\end{array}$ \\
\hline $2^{\text {nd }}$ cut & $\begin{array}{c}0.443 \\
b\end{array}$ & $\begin{array}{c}0.527 \\
\mathrm{a}\end{array}$ & $\begin{array}{c}0.582 \\
a\end{array}$ & $\begin{array}{c}0.563 \\
\mathrm{a}\end{array}$ & $\begin{array}{c}0.857 \\
\mathrm{ab}\end{array}$ & $\begin{array}{c}0.910 \\
\mathrm{a}\end{array}$ & $\begin{array}{c}0.904 \\
\mathrm{ab}\end{array}$ & $\begin{array}{c}0.823 \\
b\end{array}$ \\
\hline $3^{\text {rd }}$ cut & $\begin{array}{c}0.095 \\
\mathrm{c}\end{array}$ & $\begin{array}{c}0.115 \\
b\end{array}$ & $\begin{array}{c}0.138 \\
\mathrm{a}\end{array}$ & $\begin{array}{c}0.096 \\
\mathrm{c}\end{array}$ & $\begin{array}{c}0.330 \\
\mathrm{a}\end{array}$ & $\begin{array}{c}0.328 \\
\mathrm{a}\end{array}$ & $\begin{array}{c}0.311 \\
\mathrm{a}\end{array}$ & $\begin{array}{c}0.293 \\
\mathrm{a}\end{array}$ \\
\hline
\end{tabular}

Egypt. J. Agron. 36, No. 1 (2014) 
FORAGE GROWTH AND PRODUCTIVITY...

TABLE 6. Effect of the interaction between saline water irrigation levels and sowing date on some growth characters and forage yield of pearl millet (average the two seasons 2011 and 2012).

\begin{tabular}{|c|c|c|c|c|c|c|}
\hline Saline water & \multicolumn{3}{|c|}{4500 ppm } & \multicolumn{3}{|c|}{ 8000ppm } \\
\hline Planting date & $1 / 5$ & $15 / 5$ & $1 / 6$ & $1 / 5$ & $15 / 5$ & $1 / 6$ \\
\hline \multicolumn{7}{|c|}{$1^{\text {st }}$ cut } \\
\hline $\begin{array}{l}\text { Plant height } \\
(\mathrm{cm})\end{array}$ & $\begin{array}{c}95.72 \\
b c\end{array}$ & $\begin{array}{c}98.06 \\
b\end{array}$ & $\begin{array}{c}104.5 \\
\mathrm{a}\end{array}$ & $\begin{array}{c}88.64 \\
\mathrm{~d}\end{array}$ & $\begin{array}{c}92.91 \\
\text { cd }\end{array}$ & $\begin{array}{c}90.96 \\
\mathrm{~d}\end{array}$ \\
\hline No. of tiller $/ \mathrm{m}^{2}$ & $\begin{array}{c}125.8 \\
\mathrm{a}\end{array}$ & $\begin{array}{c}112.9 \\
b\end{array}$ & $\begin{array}{c}120.6 \\
\mathrm{a}\end{array}$ & $\begin{array}{c}84.65 \\
c\end{array}$ & $\begin{array}{c}63.55 \\
\mathrm{~d}\end{array}$ & $\begin{array}{c}54.20 \\
\mathrm{e}\end{array}$ \\
\hline $\mathrm{LA} / \mathrm{m}^{2}$ & $\begin{array}{c}79130 \\
b\end{array}$ & $\begin{array}{c}66690 \\
c\end{array}$ & $\begin{array}{c}87020 \\
\text { a }\end{array}$ & $\begin{array}{c}15310 \\
\mathrm{~d}\end{array}$ & $\begin{array}{c}11370 \\
\mathrm{~d}\end{array}$ & $\begin{array}{c}11480 \\
\mathrm{~d}\end{array}$ \\
\hline LAI & $\begin{array}{c}7.913 \\
b\end{array}$ & $\begin{array}{c}6.669 \\
c\end{array}$ & $\begin{array}{c}8.702 \\
a\end{array}$ & $\begin{array}{c}1.531 \\
\mathrm{~d}\end{array}$ & $\begin{array}{c}1.137 \\
\mathrm{~d}\end{array}$ & $\begin{array}{c}1.148 \\
\mathrm{~d}\end{array}$ \\
\hline $\begin{array}{l}\text { Total plant dry } \\
\text { (ton/fed) }\end{array}$ & $\begin{array}{c}0.725 \\
b\end{array}$ & $\begin{array}{c}0.769 \\
b\end{array}$ & $\begin{array}{c}0.868 \\
\mathrm{a}\end{array}$ & $\begin{array}{c}0.480 \\
\mathrm{c}\end{array}$ & $\begin{array}{c}0.535 \\
\mathrm{c}\end{array}$ & $\begin{array}{c}0.453 \\
\mathrm{c}\end{array}$ \\
\hline $\begin{array}{l}\text { Leaves dry } \\
\text { weight (ton/fed) }\end{array}$ & $\begin{array}{c}0.455 \\
\mathrm{a}\end{array}$ & $\begin{array}{c}0.472 \\
\mathrm{a}\end{array}$ & $\begin{array}{c}0.496 \\
\mathrm{a}\end{array}$ & $\begin{array}{c}0.260 \\
\mathrm{c}\end{array}$ & $\begin{array}{c}0.305 \\
b\end{array}$ & $\begin{array}{c}0.215 \\
\mathrm{~d}\end{array}$ \\
\hline $\begin{array}{l}\text { Stem dry } \\
\text { weight (ton/fed) }\end{array}$ & $\begin{array}{c}0.271 \\
\mathrm{bc}\end{array}$ & $\begin{array}{c}0.297 \\
b\end{array}$ & $\begin{array}{c}0.372 \\
\mathrm{a}\end{array}$ & $\begin{array}{c}0.215 \\
\mathrm{~d}\end{array}$ & $\begin{array}{c}0.230 \\
\mathrm{~cd}\end{array}$ & $\begin{array}{c}0.239 \\
\mathrm{~cd}\end{array}$ \\
\hline $\begin{array}{l}\text { Leaves/stem } \\
\text { ratio } \%\end{array}$ & $\begin{array}{c}1.699 \\
\mathrm{a}\end{array}$ & $\begin{array}{c}1.607 \\
\mathrm{a}\end{array}$ & $\begin{array}{c}1.368 \\
\mathrm{~b}\end{array}$ & $\begin{array}{c}1.203 \\
\mathrm{c}\end{array}$ & $\begin{array}{c}1.326 \\
\mathrm{bc}\end{array}$ & $\begin{array}{c}0.939 \\
\mathrm{~d}\end{array}$ \\
\hline \multicolumn{7}{|c|}{$2^{n d}$ cut } \\
\hline $\begin{array}{l}\text { Plant height } \\
(\mathrm{cm})\end{array}$ & $\begin{array}{c}122.4 \\
\mathrm{a}\end{array}$ & $\begin{array}{c}117.7 \\
b\end{array}$ & $\begin{array}{c}114.3 \\
\mathrm{~b}\end{array}$ & $\begin{array}{c}98.69 \\
\mathrm{c}\end{array}$ & $\begin{array}{c}95.05 \\
\mathrm{c}\end{array}$ & $\begin{array}{l}0 \\
\mathrm{~d}\end{array}$ \\
\hline No. of tiller $/ \mathrm{m}^{2}$ & $\begin{array}{c}106.8 \\
\mathrm{a}\end{array}$ & $\begin{array}{c}93.65 \\
b\end{array}$ & $\begin{array}{c}74.95 \\
\mathrm{c}\end{array}$ & $\begin{array}{c}34.87 \\
\mathrm{e}\end{array}$ & $\begin{array}{c}46.30 \\
\mathrm{~d}\end{array}$ & $\begin{array}{l}0 \\
\mathrm{f}\end{array}$ \\
\hline $\mathrm{LA} / \mathrm{m}^{2}$ & $\begin{array}{c}94620 \\
\text { a }\end{array}$ & $\begin{array}{c}68040 \\
\text { b }\end{array}$ & $\begin{array}{c}22540 \\
\mathrm{c}\end{array}$ & $\begin{array}{c}7207 \\
d\end{array}$ & $\begin{array}{c}5173 \\
\mathrm{~d}\end{array}$ & $\begin{array}{l}0 \\
\mathrm{e}\end{array}$ \\
\hline LAI & $\begin{array}{c}9.462 \\
\mathrm{a}\end{array}$ & $\begin{array}{c}6.804 \\
\mathrm{~b}\end{array}$ & $\begin{array}{c}2.254 \\
\mathrm{c}\end{array}$ & $\begin{array}{c}0.721 \\
\mathrm{~d}\end{array}$ & $\begin{array}{c}0.517 \\
\mathrm{~d}\end{array}$ & $\begin{array}{l}0 \\
\mathrm{e}\end{array}$ \\
\hline $\begin{array}{l}\text { Total plant dry } \\
\text { (ton/fed) }\end{array}$ & $\begin{array}{c}0.848 \\
\mathrm{a}\end{array}$ & $\begin{array}{c}0.823 \\
\mathrm{a}\end{array}$ & $\begin{array}{c}0.609 \\
b\end{array}$ & $\begin{array}{c}0.444 \\
\mathrm{c}\end{array}$ & $\begin{array}{c}0.449 \\
\mathrm{c}\end{array}$ & $\begin{array}{l}0 \\
\mathrm{~d}\end{array}$ \\
\hline $\begin{array}{l}\text { Leaves dry } \\
\text { weight (ton/fed) }\end{array}$ & $\begin{array}{c}0.476 \\
\mathrm{a}\end{array}$ & $\begin{array}{c}0.442 \\
\mathrm{a}\end{array}$ & $\begin{array}{c}0.300 \\
b\end{array}$ & $\begin{array}{c}0.192 \\
\mathrm{c}\end{array}$ & $\begin{array}{c}0.224 \\
\mathrm{c}\end{array}$ & $\begin{array}{l}0 \\
\mathrm{~d}\end{array}$ \\
\hline $\begin{array}{l}\text { Stem dry } \\
\text { weight (ton/fed) }\end{array}$ & $\begin{array}{c}0.373 \\
\mathrm{a}\end{array}$ & $\begin{array}{c}0.377 \\
\mathrm{a}\end{array}$ & $\begin{array}{c}0.312 \\
b\end{array}$ & $\begin{array}{c}0.251 \\
\mathrm{c}\end{array}$ & $\begin{array}{c}0.226 \\
\mathrm{c}\end{array}$ & $\begin{array}{l}0 \\
\mathrm{~d}\end{array}$ \\
\hline $\begin{array}{l}\text { Leaves/stem } \\
\text { ratio } \%\end{array}$ & $\begin{array}{c}1.291 \\
\mathrm{a}\end{array}$ & $\begin{array}{c}1.192 \\
\mathrm{a}\end{array}$ & $\begin{array}{c}0.973 \\
\mathrm{~b}\end{array}$ & $\begin{array}{c}0.789 \\
\mathrm{c}\end{array}$ & $\begin{array}{c}0.997 \\
b\end{array}$ & $\begin{array}{l}0 \\
\mathrm{~d}\end{array}$ \\
\hline \multicolumn{7}{|c|}{$3^{r d}$ cut } \\
\hline $\begin{array}{l}\text { Plant height } \\
(\mathrm{cm})\end{array}$ & $\begin{array}{c}122.4 \\
\mathrm{a}\end{array}$ & $\begin{array}{c}114.1 \\
\mathrm{~b}\end{array}$ & $\begin{array}{l}0 \\
\mathrm{c}\end{array}$ & $\begin{array}{l}0 \\
\mathrm{c}\end{array}$ & $\begin{array}{l}0 \\
\mathrm{c}\end{array}$ & $\begin{array}{l}0 \\
\mathrm{c}\end{array}$ \\
\hline No. of tiller $/ \mathrm{m}^{2}$ & $\begin{array}{c}32.75 \\
\mathrm{a}\end{array}$ & $\begin{array}{c}26.78 \\
b\end{array}$ & $\begin{array}{l}0 \\
\mathrm{c}\end{array}$ & $\begin{array}{l}0 \\
\mathrm{c}\end{array}$ & $\begin{array}{l}0 \\
\mathrm{c}\end{array}$ & $\begin{array}{l}0 \\
\mathrm{c}\end{array}$ \\
\hline $\mathrm{LA}\left(\mathrm{cm}^{2} / \mathrm{m}^{2}\right)$ & $\begin{array}{c}12660 \\
\text { a }\end{array}$ & $\begin{array}{c}11630 \\
b\end{array}$ & $\begin{array}{l}0 \\
\mathrm{c}\end{array}$ & $\begin{array}{l}0 \\
\mathrm{c}\end{array}$ & $\begin{array}{l}0 \\
\mathrm{c}\end{array}$ & $\begin{array}{l}0 \\
\mathrm{c}\end{array}$ \\
\hline LAI & $\begin{array}{c}1.266 \\
\mathrm{a}\end{array}$ & $\begin{array}{c}1.163 \\
b\end{array}$ & $\begin{array}{l}0 \\
\mathrm{c}\end{array}$ & $\begin{array}{l}0 \\
\mathrm{c}\end{array}$ & $\begin{array}{l}0 \\
\mathrm{c}\end{array}$ & $\begin{array}{l}0 \\
\mathrm{c}\end{array}$ \\
\hline $\begin{array}{l}\text { Total plant dry } \\
\text { (ton/fed) }\end{array}$ & $\begin{array}{c}0.408 \\
\mathrm{a}\end{array}$ & $\begin{array}{c}0.259 \\
\mathrm{~b}\end{array}$ & $\begin{array}{l}0 \\
\mathrm{c}\end{array}$ & $\begin{array}{l}0 \\
\mathrm{c}\end{array}$ & $\begin{array}{l}0 \\
\mathrm{c}\end{array}$ & $\begin{array}{l}0 \\
\mathrm{c}\end{array}$ \\
\hline $\begin{array}{l}\text { Leaves dry } \\
\text { weight (ton/fed) }\end{array}$ & $\begin{array}{c}0.194 \\
\mathrm{a}\end{array}$ & $\begin{array}{c}0.125 \\
b\end{array}$ & $\begin{array}{l}0 \\
\mathrm{c}\end{array}$ & $\begin{array}{l}0 \\
\mathrm{c}\end{array}$ & $\begin{array}{l}0 \\
\mathrm{c}\end{array}$ & $\begin{array}{l}0 \\
\mathrm{c}\end{array}$ \\
\hline $\begin{array}{l}\text { Stem dry } \\
\text { weight (ton/fed) }\end{array}$ & $\begin{array}{c}0.205 \\
\mathrm{a}\end{array}$ & $\begin{array}{c}0.134 \\
b\end{array}$ & $\begin{array}{l}0 \\
\mathrm{c}\end{array}$ & $\begin{array}{l}0 \\
\mathrm{c}\end{array}$ & $\begin{array}{l}0 \\
\mathrm{c}\end{array}$ & $\begin{array}{l}0 \\
\mathrm{c}\end{array}$ \\
\hline $\begin{array}{l}\text { Leaves/stem } \\
\text { ratio }\end{array}$ & $\begin{array}{c}0.955 \\
\mathrm{a}\end{array}$ & $\begin{array}{c}0.937 \\
\mathrm{a}\end{array}$ & $\begin{array}{l}0 \\
\mathrm{~b}\end{array}$ & $\begin{array}{l}0 \\
b\end{array}$ & $\begin{array}{l}0 \\
b\end{array}$ & $\begin{array}{l}0 \\
b\end{array}$ \\
\hline
\end{tabular}

Egypt. J. Agron. 36, No. 1 (2014) 
In addition to the above results, under high saline water irrigation level $8000 \mathrm{ppm}$ there was no any re-growth of the pearl millet plants in the three sowing dates, i.e. $1^{\text {st }}$ May, $15^{\text {th }}$ May and $1^{\text {st }}$ June. This is may be due to the combine harmful effect of the high salinity of the irrigation water and the high temperature on the re-growth of the plants.

Results in Table 7 showed the effect of the interaction between irrigation water salinity and soil mulching on the forage yield and some growth characters of pearl millet. Obtained data showed that at the $1^{\text {st }}$ cut using plastic soil mulching produced the significant values of plant height, number of tillers, leaves area $/ \mathrm{m}^{2}$, leaf area index, total plant dry weight, leaves dry weight, stem dry weight and leaves/stem ratio under the irrigation water salinity $4500 \mathrm{ppm}$. Whereas, using plastic for soil mulching produced the lowest significant values of number of tillers, leaves area $/ \mathrm{m}^{2}$ and leaf area index under the high saline water irrigation $8000 \mathrm{ppm}$.

At the $2^{\text {nd }}$ cut results showed that, using 1 ton of rice straw for soil mulching produced the significant values of tillers number, leaves area $/ \mathrm{m}^{2}$ and leaf area index under the low saline irrigation water $(4500 \mathrm{ppm})$. Whereas, using 2 ton of rice straw treatment produced the high significant values of the total plant dry weight, leaves dry weight, stem dry weight and leaves/stem ratio under the irrigation water salinity $4500 \mathrm{ppm}$ conditions. In the main time, the same soil mulching treatment produced the lowest significantly values of the total plant dry weight, leaves dry weight and stem dry weight under the irrigation water salinity 8000 ppm treatment.

At the $3^{\text {rd }}$ cut there was no re-growth of pearl millet under the high saline irrigation water $(8000 \mathrm{ppm})$. Results obtained showed that using plastic treatment produced the high significant values of plant height, number of tillers, total plant dry weight, leaves dry weight and stem dry weight under salinity irrigation water $4500 \mathrm{ppm}$ treatment. Generally results indicated that the same soil mulching material or the amount of the material does not has the same effect on the pearl millet yield under different saline water irrigation levels. The results indicated that under the low irrigation water salinity using plastic for soil mulching is more efficiency than using rice straw, whereas, the reverse is true under the high irrigation water salinity conditions.

Results in Table 8 showed that at the $1^{\text {st }}$ cut using plastic produced the significant values of plant height, total plant dry weight at sowing date $15^{\text {th }}$ May, leaves area $/ \mathrm{m}^{2}$, leaf area index and stem dry weight at sowing date $1^{\text {st }}$ June. The higher significant value of leaves/stem ratio was obtained by using 2 ton of rice straw at sowing date of the $1^{\text {st }}$ May.

At the $2^{\text {nd }}$ cut there were no significant differences among the soil mulching materials and amounts in the total plant dry weight at sowing date of $1^{\text {st }}$ May and $15^{\text {th }}$ May. On the other hand, using 2 ton of rice straw for soil mulching had the significant value of leaves dry weight at sowing date $15^{\text {th }}$ May and the high stem dry weight at sowing date $1^{\text {st }}$ May which produced the significant value of number of tillers at this sowing date.

Egypt. J. Agron. 36, No. 1 (2014) 
TABLE 7. Effect of the interaction between saline water irrigation levels and soil mulching on some growth characters and forage yield of pearl millet (average the two seasons 2011 and 2012).

\begin{tabular}{|c|c|c|c|c|c|c|c|c|}
\hline Saline water & \multicolumn{4}{|c|}{$4500 \mathrm{ppm}$} & \multicolumn{4}{|c|}{ 8000ppm } \\
\hline $\begin{array}{c}\text { Soil } \\
\text { mulching }\end{array}$ & Without & $\begin{array}{l}1 \text { ton/ } \\
\text { fed } \\
\text { straw }\end{array}$ & $\begin{array}{l}2 \text { ton/ } \\
\text { fed } \\
\text { straw }\end{array}$ & plastic & Without & $\begin{array}{l}1 \text { ton/ } \\
\text { fed } \\
\text { straw }\end{array}$ & $\begin{array}{c}2 \text { ton/ } \\
\text { fed } \\
\text { straw }\end{array}$ & plastic \\
\hline \multicolumn{9}{|c|}{$1^{s t} \mathrm{cut}$} \\
\hline $\begin{array}{l}\text { Plant height } \\
(\mathrm{cm})\end{array}$ & $\begin{array}{c}99.11 \\
\mathrm{ab}\end{array}$ & $\begin{array}{c}97.94 \\
b\end{array}$ & $\begin{array}{c}97.99 \\
\text { b }\end{array}$ & $\begin{array}{c}102.7 \\
\mathrm{a}\end{array}$ & $\begin{array}{c}87.26 \\
d\end{array}$ & $\begin{array}{c}91.87 \\
\mathrm{c}\end{array}$ & $\begin{array}{c}90.36 \\
\text { cd }\end{array}$ & $\begin{array}{c}93.86 \\
\mathrm{c}\end{array}$ \\
\hline No of tiller $/ \mathrm{m}^{2}$ & $\begin{array}{c}121.9 \\
\mathrm{ab}\end{array}$ & $\begin{array}{c}117.5 \\
\text { bc }\end{array}$ & $\begin{array}{c}113.1 \\
\mathrm{c}\end{array}$ & $\begin{array}{c}126.5 \\
a\end{array}$ & $\begin{array}{c}71.87 \\
d\end{array}$ & $\begin{array}{c}69.20 \\
\mathrm{~d}\end{array}$ & $\begin{array}{c}66.07 \\
\text { de }\end{array}$ & $\begin{array}{c}62.73 \\
\mathrm{e}\end{array}$ \\
\hline $\mathrm{LA}\left(\mathrm{cm}^{2} / \mathrm{m}^{2}\right)$ & $\begin{array}{c}74340 \\
\text { bc }\end{array}$ & $\begin{array}{c}80110 \\
\mathrm{ab}\end{array}$ & $\begin{array}{c}70890 \\
c\end{array}$ & $\begin{array}{c}85110 \\
a\end{array}$ & $\begin{array}{c}13260 \\
\mathrm{~d}\end{array}$ & $\begin{array}{c}12270 \\
\mathrm{~d}\end{array}$ & $\begin{array}{c}13580 \\
\mathrm{~d}\end{array}$ & $\begin{array}{c}11760 \\
d\end{array}$ \\
\hline LAI & $\begin{array}{c}7.434 \\
\text { bc }\end{array}$ & $\begin{array}{c}8.011 \\
\mathrm{ab}\end{array}$ & $\begin{array}{c}7.089 \\
\mathrm{c}\end{array}$ & $\begin{array}{c}8.511 \\
\mathrm{a}\end{array}$ & $\begin{array}{c}1.326 \\
\mathrm{~d}\end{array}$ & $\begin{array}{c}1.227 \\
\mathrm{~d}\end{array}$ & $\begin{array}{c}1.358 \\
\mathrm{~d}\end{array}$ & $\begin{array}{c}1.176 \\
\mathrm{~d}\end{array}$ \\
\hline $\begin{array}{l}\text { Total plant } \\
\text { dry (ton/fed) }\end{array}$ & $\begin{array}{c}0.800 \\
b\end{array}$ & $\begin{array}{c}0.734 \\
b\end{array}$ & $\begin{array}{c}0.690 \\
b\end{array}$ & $\begin{array}{c}0.924 \\
\mathrm{a}\end{array}$ & $\begin{array}{c}0.473 \\
\mathrm{c}\end{array}$ & $\begin{array}{c}0.488 \\
\mathrm{c}\end{array}$ & $\begin{array}{c}0.468 \\
\mathrm{c}\end{array}$ & $\begin{array}{c}0.529 \\
\mathrm{c}\end{array}$ \\
\hline $\begin{array}{l}\text { Leaves dry } \\
\text { weight (ton/fed) }\end{array}$ & $\begin{array}{c}0.482 \\
b\end{array}$ & $\begin{array}{c}0.447 \\
b\end{array}$ & $\begin{array}{c}0.425 \\
b\end{array}$ & $\begin{array}{c}0.543 \\
\mathrm{a}\end{array}$ & $\begin{array}{c}0.250 \\
\mathrm{c}\end{array}$ & $\begin{array}{c}0.256 \\
\mathrm{c}\end{array}$ & $\begin{array}{c}0.256 \\
\mathrm{c}\end{array}$ & $\begin{array}{c}0.278 \\
\mathrm{c}\end{array}$ \\
\hline $\begin{array}{l}\text { Stem dry } \\
\text { weight (ton/fed) }\end{array}$ & $\begin{array}{c}0.319 \\
b\end{array}$ & $\begin{array}{c}0.287 \\
\text { bc }\end{array}$ & $\begin{array}{c}0.266 \\
\text { bcd }\end{array}$ & $\begin{array}{c}0.380 \\
\mathrm{a}\end{array}$ & $\begin{array}{c}0.223 \\
\mathrm{~cd}\end{array}$ & $\begin{array}{c}0.230 \\
\mathrm{~cd}\end{array}$ & $\begin{array}{c}0.212 \\
\mathrm{~d}\end{array}$ & $\begin{array}{c}0.247 \\
\mathrm{~cd}\end{array}$ \\
\hline $\begin{array}{l}\text { Leaves/stem } \\
\text { ratio } \%\end{array}$ & $\begin{array}{c}1.567 \\
\mathrm{a}\end{array}$ & $\begin{array}{c}1.573 \\
\mathrm{a}\end{array}$ & $\begin{array}{c}1.623 \\
\mathrm{a}\end{array}$ & $\begin{array}{c}1.470 \\
\mathrm{a}\end{array}$ & $\begin{array}{c}1.136 \\
b\end{array}$ & $\begin{array}{c}1.126 \\
b\end{array}$ & $\begin{array}{c}1.205 \\
b\end{array}$ & $\begin{array}{c}1.156 \\
b\end{array}$ \\
\hline \multicolumn{9}{|c|}{$2^{\text {nd }}$ cut } \\
\hline $\begin{array}{l}\text { Plant height } \\
\text { (cm) }\end{array}$ & $\begin{array}{c}116.4 \\
b\end{array}$ & $\begin{array}{c}111.5 \\
\mathrm{c}\end{array}$ & $\begin{array}{c}120.7 \\
a\end{array}$ & $\begin{array}{c}124.0 \\
\mathrm{a}\end{array}$ & $\begin{array}{c}61.55 \\
\mathrm{e}\end{array}$ & $\begin{array}{c}62.82 \\
\mathrm{e}\end{array}$ & $\begin{array}{c}67.42 \\
\mathrm{~d}\end{array}$ & $\begin{array}{c}66.38 \\
\mathrm{~d}\end{array}$ \\
\hline No of tiller $/ \mathrm{m}^{2}$ & $\begin{array}{c}80.17 \\
\mathrm{c}\end{array}$ & $\begin{array}{c}101.5 \\
\mathrm{a}\end{array}$ & $\begin{array}{c}94.97 \\
\mathrm{ab}\end{array}$ & $\begin{array}{c}90.57 \\
b\end{array}$ & $\begin{array}{c}29.17 \\
\mathrm{~d}\end{array}$ & $\begin{array}{c}25.10 \\
\mathrm{~d}\end{array}$ & $\begin{array}{c}26.67 \\
d\end{array}$ & $\begin{array}{c}27.30 \\
d\end{array}$ \\
\hline $\mathrm{LA}\left(\mathrm{cm}^{2} / \mathrm{m}^{2}\right)$ & $\begin{array}{c}60800 \\
b\end{array}$ & $\begin{array}{c}67440 \\
a\end{array}$ & $\begin{array}{c}65570 \\
\mathrm{ab}\end{array}$ & $\begin{array}{c}53130 \\
\mathrm{c}\end{array}$ & $\begin{array}{c}3762 \\
d\end{array}$ & $\begin{array}{c}4623 \\
d\end{array}$ & $\begin{array}{c}5011 \\
\mathrm{~d}\end{array}$ & $\begin{array}{c}3110 \\
\mathrm{~d}\end{array}$ \\
\hline LAI & $\begin{array}{c}6.080 \\
b\end{array}$ & $\begin{array}{c}6.744 \\
\mathrm{a}\end{array}$ & $\begin{array}{c}6.557 \\
\mathrm{ab}\end{array}$ & $\begin{array}{c}5.313 \\
\mathrm{c}\end{array}$ & $\begin{array}{c}0.376 \\
\mathrm{~d}\end{array}$ & $\begin{array}{c}0.462 \\
\mathrm{~d}\end{array}$ & $\begin{array}{c}0.501 \\
\mathrm{~d}\end{array}$ & $\begin{array}{c}0.311 \\
\mathrm{~d}\end{array}$ \\
\hline $\begin{array}{l}\text { Total plant } \\
\text { dry (ton/fed) }\end{array}$ & $\begin{array}{c}0.663 \\
b\end{array}$ & $\begin{array}{c}0.773 \\
\mathrm{a}\end{array}$ & $\begin{array}{c}0.832 \\
\mathrm{a}\end{array}$ & $\begin{array}{c}0.772 \\
\mathrm{a}\end{array}$ & $\begin{array}{c}0.223 \\
\mathrm{~d}\end{array}$ & $\begin{array}{c}0.282 \\
\text { cd }\end{array}$ & $\begin{array}{c}0.332 \\
\mathrm{c}\end{array}$ & $\begin{array}{c}0.355 \\
\mathrm{c}\end{array}$ \\
\hline $\begin{array}{l}\text { Leaves dry } \\
\text { weight (ton/fed) }\end{array}$ & $\begin{array}{c}0.348 \\
\mathrm{c}\end{array}$ & $\begin{array}{c}0.413 \\
b\end{array}$ & $\begin{array}{c}0.458 \\
\mathrm{a}\end{array}$ & $\begin{array}{c}0.405 \\
b\end{array}$ & $\begin{array}{c}0.106 \\
\mathrm{e}\end{array}$ & $\begin{array}{c}0.140 \\
\mathrm{~d}\end{array}$ & $\begin{array}{c}0.148 \\
\mathrm{~d}\end{array}$ & $\begin{array}{c}0.160 \\
\mathrm{~d}\end{array}$ \\
\hline $\begin{array}{l}\text { Stem dry } \\
\text { weight }(\tan / \mathrm{fed})\end{array}$ & $\begin{array}{c}0.316 \\
\mathrm{~b}\end{array}$ & $\begin{array}{c}0.355 \\
\mathrm{ab}\end{array}$ & $\begin{array}{c}0.374 \\
\mathrm{a}\end{array}$ & $\begin{array}{c}0.371 \\
\mathrm{a}\end{array}$ & $\begin{array}{c}0.117 \\
\mathrm{e}\end{array}$ & $\begin{array}{c}0.142 \\
\text { de }\end{array}$ & $\begin{array}{c}0.183 \\
\text { cd }\end{array}$ & $\begin{array}{c}0.193 \\
\mathrm{c}\end{array}$ \\
\hline $\begin{array}{l}\text { Leaves/stem } \\
\text { ratio } \%\end{array}$ & $\begin{array}{c}1.101 \\
b\end{array}$ & $\begin{array}{c}1.166 \\
\mathrm{ab}\end{array}$ & $\begin{array}{c}1.255 \\
\mathrm{a}\end{array}$ & $\begin{array}{c}1.087 \\
\mathrm{~b}\end{array}$ & $\begin{array}{c}0.613 \\
\mathrm{c}\end{array}$ & $\begin{array}{c}0.654 \\
\mathrm{c}\end{array}$ & $\begin{array}{c}0.554 \\
\mathrm{c}\end{array}$ & $\begin{array}{c}0.560 \\
\mathrm{c}\end{array}$ \\
\hline \multicolumn{9}{|c|}{$3^{r a}$ cut } \\
\hline $\begin{array}{l}\begin{array}{l}\text { Plant height } \\
(\mathrm{cm})\end{array} \\
\end{array}$ & $\begin{array}{c}71.96 \\
\mathrm{~d}\end{array}$ & $\begin{array}{c}75.83 \\
c\end{array}$ & $\begin{array}{c}81.55 \\
b\end{array}$ & $\begin{array}{c}86.02 \\
\mathrm{a}\end{array}$ & $\begin{array}{l}0 \\
\mathrm{e}\end{array}$ & $\begin{array}{l}0 \\
\mathrm{e}\end{array}$ & $\begin{array}{l}0 \\
\mathrm{e}\end{array}$ & $\begin{array}{l}0 \\
\mathrm{e}\end{array}$ \\
\hline No of tiller $/ \mathrm{m}^{2}$ & $\begin{array}{c}20.37 \\
\mathrm{a}\end{array}$ & $\begin{array}{c}18.87 \\
b\end{array}$ & $\begin{array}{c}19.80 \\
\mathrm{ab}\end{array}$ & $\begin{array}{c}20.33 \\
\mathrm{a}\end{array}$ & $\begin{array}{l}0 \\
\mathrm{c}\end{array}$ & $\begin{array}{l}0 \\
\mathrm{c}\end{array}$ & $\begin{array}{l}0 \\
\mathrm{c}\end{array}$ & $\begin{array}{l}0 \\
\mathrm{c}\end{array}$ \\
\hline $\mathrm{LA}\left(\mathrm{cm}^{2} / \mathrm{m}^{2}\right)$ & $\begin{array}{c}7291 \\
b\end{array}$ & $\begin{array}{c}8193 \\
a b\end{array}$ & $\begin{array}{c}9109 \\
\mathrm{a}\end{array}$ & $\begin{array}{c}7799 \\
\text { b }\end{array}$ & $\begin{array}{l}0 \\
\mathrm{c}\end{array}$ & $\begin{array}{l}0 \\
\mathrm{c}\end{array}$ & $\begin{array}{l}0 \\
\mathrm{c}\end{array}$ & $\begin{array}{l}0 \\
\mathrm{c}\end{array}$ \\
\hline LAI & $\begin{array}{c}0.729 \\
b\end{array}$ & $\begin{array}{c}0.819 \\
\mathrm{ab}\end{array}$ & $\begin{array}{c}0.911 \\
\mathrm{a}\end{array}$ & $\begin{array}{c}0.780 \\
b\end{array}$ & $\begin{array}{l}0 \\
\mathrm{c}\end{array}$ & $\begin{array}{l}0 \\
\mathrm{c}\end{array}$ & $\begin{array}{l}0 \\
\mathrm{c}\end{array}$ & $\begin{array}{l}0 \\
\mathrm{c}\end{array}$ \\
\hline $\begin{array}{l}\text { Total plant } \\
\text { dry (tan/fed) }\end{array}$ & $\begin{array}{c}0.191 \\
\mathrm{c}\end{array}$ & $\begin{array}{c}0.230 \\
b\end{array}$ & $\begin{array}{c}0.277 \\
\mathrm{a}\end{array}$ & $\begin{array}{c}0.192 \\
\mathrm{c}\end{array}$ & $\begin{array}{l}0 \\
\mathrm{~d}\end{array}$ & $\begin{array}{l}0 \\
\mathrm{~d}\end{array}$ & $\begin{array}{l}0 \\
\mathrm{~d}\end{array}$ & $\begin{array}{l}0 \\
\mathrm{~d}\end{array}$ \\
\hline $\begin{array}{l}\text { Leaves dry } \\
\text { weight }(\tan / \mathrm{fed})\end{array}$ & $\begin{array}{c}0.094 \\
\mathrm{c}\end{array}$ & $\begin{array}{c}0.111 \\
b\end{array}$ & $\begin{array}{c}0.130 \\
\mathrm{a}\end{array}$ & $\begin{array}{c}0.090 \\
\mathrm{c}\end{array}$ & $\begin{array}{l}0 \\
\mathrm{~d}\end{array}$ & $\begin{array}{l}0 \\
\mathrm{~d}\end{array}$ & d & $\begin{array}{l}0 \\
\mathrm{~d}\end{array}$ \\
\hline $\begin{array}{l}\text { Stem dry } \\
\text { weight }(\tan / \text { fed })\end{array}$ & $\begin{array}{c}0.097 \\
\mathrm{c}\end{array}$ & $\begin{array}{c}0.114 \\
b\end{array}$ & $\begin{array}{c}0.140 \\
\mathrm{a}\end{array}$ & $\begin{array}{c}0.102 \\
\mathrm{c}\end{array}$ & $\begin{array}{l}0 \\
\mathrm{~d}\end{array}$ & $\begin{array}{l}0 \\
\mathrm{~d}\end{array}$ & $\begin{array}{l}0 \\
\mathrm{~d}\end{array}$ & $\begin{array}{l}0 \\
\mathrm{~d}\end{array}$ \\
\hline
\end{tabular}




\begin{tabular}{|l|c|c|c|c|c|c|l|l|}
\hline $\begin{array}{l}\text { Leaves/stem } \\
\text { ratio \% }\end{array}$ & $\begin{array}{c}0.659 \\
\mathrm{a}\end{array}$ & $\begin{array}{c}0.656 \\
\mathrm{a}\end{array}$ & $\begin{array}{c}0.622 \\
\mathrm{ab}\end{array}$ & $\begin{array}{c}0.586 \\
\mathrm{~b}\end{array}$ & $\begin{array}{c}0 \\
\mathrm{c}\end{array}$ & $\begin{array}{l}0 \\
\mathrm{c}\end{array}$ & $\begin{array}{l}0 \\
\mathrm{c}\end{array}$ & $\begin{array}{l}0 \\
\mathrm{c}\end{array}$ \\
\hline
\end{tabular}

TABLE 8. Effect of the interaction between saline water irrigation levels and soil mulching on some growth characters and forage yield of pearl millet (average the two seasons 2011 and 2012).

\begin{tabular}{|c|c|c|c|c|c|c|c|c|c|c|c|c|}
\hline \multirow{2}{*}{$\begin{array}{c}\text { Plant date } \\
\begin{array}{c}\text { Soil } \\
\text { mulching }\end{array}\end{array}$} & \multicolumn{4}{|c|}{\begin{tabular}{|r|r|}
$1 / 5$ \\
\end{tabular}} & \multicolumn{4}{|c|}{$15 / 5$} & \multicolumn{4}{|c|}{$1 / 6$} \\
\hline & Without & $\begin{array}{l}\text { 1ton/ } \\
\text { fed } \\
\text { straw }\end{array}$ & \begin{tabular}{|c|} 
2ton/ \\
fed \\
straw
\end{tabular} & plastic & Without & $\begin{array}{l}\text { 1ton/ } \\
\text { fed } \\
\text { straw }\end{array}$ & \begin{tabular}{|c} 
2ton/ \\
fed \\
straw
\end{tabular} & plastic & Without & $\begin{array}{l}\text { 1ton/ } \\
\text { fed } \\
\text { straw }\end{array}$ & \begin{tabular}{|c}
$\begin{array}{c}\text { 2ton/ } \\
\text { fed } \\
\text { straw }\end{array}$ \\
\end{tabular} & plastic \\
\hline \multicolumn{13}{|c|}{$1^{s t}$ cut } \\
\hline $\begin{array}{l}\text { Plant height } \\
\text { (cm) }\end{array}$ & $\begin{array}{c}85.15 \\
\mathrm{e}\end{array}$ & $\begin{array}{c}94.92 \\
\text { bcd }\end{array}$ & $\begin{array}{c}94.59 \\
\mathrm{~cd}\end{array}$ & $\begin{array}{c}94.06 \\
\mathrm{~cd}\end{array}$ & $\begin{array}{c}95.47 \\
\text { bcd }\end{array}$ & $\begin{array}{c}92.68 \\
d\end{array}$ & $\begin{array}{c}92.93 \\
\mathrm{~d}\end{array}$ & $\begin{array}{c}100.9 \\
\mathrm{a}\end{array}$ & $\begin{array}{c}98.92 \\
a b c\end{array}$ & $\begin{array}{c}97.12 \\
\text { a-d }\end{array}$ & $\begin{array}{c}95.01 \\
\text { bcd }\end{array}$ & $\begin{array}{c}99.9 \\
\mathrm{ab}\end{array}$ \\
\hline $\begin{array}{l}\text { No of tiller / } \\
\mathrm{m}^{2}\end{array}$ & $\begin{array}{c}126.0 \\
\mathrm{a}\end{array}$ & $\begin{array}{c}95.05 \\
\mathrm{c}\end{array}$ & $\begin{array}{c}104.6 \\
\text { b }\end{array}$ & $\begin{array}{c}95.35 \\
\mathrm{c}\end{array}$ & $\begin{array}{c}92.45 \\
\mathrm{c}\end{array}$ & $\begin{array}{c}84.55 \\
\mathrm{~d}\end{array}$ & $\begin{array}{c}80.05 \\
\mathrm{~d}\end{array}$ & $\begin{array}{c}95.75 \\
\mathrm{c}\end{array}$ & $\begin{array}{c}72.20 \\
\mathrm{e}\end{array}$ & $\begin{array}{c}100.5 \\
b c\end{array}$ & $\begin{array}{c}84.10 \\
\mathrm{~d}\end{array}$ & $\begin{array}{c}92.75 \\
\mathrm{c}\end{array}$ \\
\hline LA $\left(\mathrm{cm}^{2} / \mathrm{m}^{2}\right)$ & $\begin{array}{c}47400 \\
\text { bcd }\end{array}$ & $\begin{array}{c}46020 \\
\text { b-e }\end{array}$ & $\begin{array}{c}49450 \\
\text { abc }\end{array}$ & $\begin{array}{c}46010 \\
\text { b-e }\end{array}$ & $\begin{array}{c}39930 \\
\text { cde }\end{array}$ & $\begin{array}{c}37720 \\
\text { de }\end{array}$ & $\begin{array}{c}36350 \\
\mathrm{e}\end{array}$ & $\begin{array}{c}42120 \\
\text { cde }\end{array}$ & $\begin{array}{c}44080 \\
\text { cde }\end{array}$ & $\begin{array}{c}54830 \\
\mathrm{ab}\end{array}$ & $\begin{array}{c}40910 \\
\text { cde }\end{array}$ & $\begin{array}{c}57170 \\
\mathrm{a} \\
\end{array}$ \\
\hline LAI & $\begin{array}{c}4.740 \\
\text { bcd }\end{array}$ & $\begin{array}{c}4.602 \\
\text { b-e }\end{array}$ & $\begin{array}{c}4.945 \\
\text { abc }\end{array}$ & $\begin{array}{c}4.601 \\
b-e\end{array}$ & $\begin{array}{c}3.993 \\
\text { cde }\end{array}$ & $\begin{array}{c}3.772 \\
\mathrm{de}\end{array}$ & $\begin{array}{c}3.635 \\
\mathrm{e}\end{array}$ & $\begin{array}{c}4.212 \\
\text { cde }\end{array}$ & $\begin{array}{c}4.408 \\
\mathrm{~cd}\end{array}$ & $\begin{array}{c}5.483 \\
a b\end{array}$ & $\begin{array}{c}4.091 \\
\text { cde }\end{array}$ & $\begin{array}{c}5.717 \\
\mathrm{a}\end{array}$ \\
\hline $\begin{array}{l}\text { Total plant } \\
\text { dry (tan/fed) }\end{array}$ & $\begin{array}{c}0.594 \\
\text { bc }\end{array}$ & $\begin{array}{c}0.557 \\
\mathrm{c}\end{array}$ & $\begin{array}{c}0.588 \\
\mathrm{bc}\end{array}$ & \begin{tabular}{|c|}
0.670 \\
$a b c$ \\
\end{tabular} & $\begin{array}{c}0.658 \\
\mathrm{abc}\end{array}$ & $\begin{array}{c}0.599 \\
\text { bc }\end{array}$ & $\begin{array}{c}0.581 \\
\text { bc }\end{array}$ & \begin{tabular}{|c|}
0.770 \\
$\mathrm{a}$
\end{tabular} & $\begin{array}{c}0.658 \\
\mathrm{abc}\end{array}$ & $\begin{array}{c}0.677 \\
a b c\end{array}$ & $\begin{array}{c}0.568 \\
\mathrm{c}\end{array}$ & \begin{tabular}{|c|}
0.740 \\
$\mathrm{ab}$ \\
\end{tabular} \\
\hline $\begin{array}{l}\text { Leaves dry } \\
\text { weight } \\
(\tan / \mathrm{fed}) \\
\end{array}$ & $\begin{array}{c}0.362 \\
\mathrm{~b}\end{array}$ & $\begin{array}{c}0.316 \\
\mathrm{~b}\end{array}$ & $\begin{array}{c}0.359 \\
b\end{array}$ & $\begin{array}{c}0.393 \\
\text { ab }\end{array}$ & $\begin{array}{c}0.394 \\
\mathrm{ab}\end{array}$ & $\begin{array}{c}0.362 \\
\mathrm{~b}\end{array}$ & $\begin{array}{c}0.445 \\
\mathrm{~b}\end{array}$ & $\begin{array}{c}0.454 \\
\mathrm{a}\end{array}$ & $\begin{array}{c}0.343 \\
\mathrm{~b}\end{array}$ & $\begin{array}{c}0.378 \\
a b\end{array}$ & $\begin{array}{c}0.317 \\
\mathrm{~b}\end{array}$ & $\begin{array}{c}0.385 \\
a b\end{array}$ \\
\hline $\begin{array}{l}\text { Stem dry } \\
\text { weight } \\
\text { (tan/fed) }\end{array}$ & $\begin{array}{c}0.233 \\
\mathrm{~b}\end{array}$ & $\begin{array}{c}0.240 \\
\mathrm{~b}\end{array}$ & $\begin{array}{c}0.229 \\
\mathrm{~b}\end{array}$ & $\begin{array}{c}0.270 \\
\mathrm{~b}\end{array}$ & $\begin{array}{c}0.264 \\
\mathrm{~b}\end{array}$ & $\begin{array}{c}0.238 \\
\mathrm{~b}\end{array}$ & $\begin{array}{c}0.236 \\
\mathrm{~b}\end{array}$ & $\begin{array}{c}0.316 \\
a b\end{array}$ & $\begin{array}{c}0.316 \\
a b\end{array}$ & $\begin{array}{c}0.299 \\
\mathrm{ab}\end{array}$ & $\begin{array}{c}0.251 \\
\mathrm{~b}\end{array}$ & $\begin{array}{c}0.355 \\
\mathrm{a}\end{array}$ \\
\hline $\begin{array}{l}\text { Leaves/stem } \\
\text { ratio }\end{array}$ & $\begin{array}{c}1.510 \\
\mathrm{ab}\end{array}$ & $\begin{array}{c}1.301 \\
\text { bcd }\end{array}$ & $\begin{array}{c}1.5644 \\
\mathrm{a}\end{array}$ & $\begin{array}{c}1.429 \\
\mathrm{abc}\end{array}$ & $\begin{array}{c}1.459 \\
\mathrm{abc}\end{array}$ & $\begin{array}{c}1.511 \\
\mathrm{ab}\end{array}$ & $\begin{array}{c}1.455 \\
\mathrm{abc}\end{array}$ & $\begin{array}{c}1.440 \\
a b c\end{array}$ & $\begin{array}{c}1.085 \\
\mathrm{~d}\end{array}$ & $\begin{array}{c}1.237 \\
\mathrm{~cd}\end{array}$ & $\begin{array}{c}1.222 \\
\mathrm{~cd}\end{array}$ & $\begin{array}{c}1.070 \\
\mathrm{~d}\end{array}$ \\
\hline \multicolumn{13}{|c|}{$2^{\text {nd }}$ cut } \\
\hline $\begin{array}{l}\text { Plant height } \\
(\mathrm{cm}) .\end{array}$ & $\begin{array}{c}103.4 \\
\text { de }\end{array}$ & $\begin{array}{c}106.4 \\
\text { bcd }\end{array}$ & $\begin{array}{c}117.2 \\
\mathrm{a}\end{array}$ & \begin{tabular}{|c|}
115.2 \\
$\mathrm{a}$ \\
\end{tabular} & $\begin{array}{c}104.9 \\
\text { cde }\end{array}$ & $\begin{array}{c}100.7 \\
\mathrm{e}\end{array}$ & $\begin{array}{c}109.2 \\
\text { bc }\end{array}$ & $\begin{array}{c}110.6 \\
\mathrm{~b}\end{array}$ & $\begin{array}{c}58.62 \\
\text { fg }\end{array}$ & $\begin{array}{c}54.36 \\
\mathrm{~g}\end{array}$ & $\begin{array}{c}55.83 \\
\text { fg }\end{array}$ & $\begin{array}{c}59.76 \\
\text { f }\end{array}$ \\
\hline $\begin{array}{l}\text { No of tiller / } \\
\mathrm{m}^{2}\end{array}$ & $\begin{array}{c}69.75 \\
a b\end{array}$ & \begin{tabular}{|c|}
74.40 \\
$\mathrm{a}$ \\
\end{tabular} & $\begin{array}{c}75.45 \\
\mathrm{a} \\
\end{array}$ & $\begin{array}{c}63.70 \\
b\end{array}$ & $\begin{array}{c}68.45 \\
a b\end{array}$ & \begin{tabular}{|c|}
70.75 \\
$a b$ \\
\end{tabular} & \begin{tabular}{|c|}
69.90 \\
$a b$ \\
\end{tabular} & \begin{tabular}{|c|}
70.80 \\
$a b$
\end{tabular} & $\begin{array}{c}25.80 \\
\mathrm{~d}\end{array}$ & $\begin{array}{c}44.70 \\
\mathrm{c}\end{array}$ & \begin{tabular}{|c|}
37.10 \\
$\mathrm{c}$
\end{tabular} & $\begin{array}{c}42.30 \\
\mathrm{c} \\
\end{array}$ \\
\hline LA $\left(\mathrm{cm}^{2} / \mathrm{m}^{2}\right)$ & $\begin{array}{c}50100 \\
\mathrm{~b}\end{array}$ & $\begin{array}{c}58490 \\
\mathrm{a}\end{array}$ & $\begin{array}{c}49780 \\
\text { b }\end{array}$ & $\begin{array}{c}45270 \\
\text { bc }\end{array}$ & $\begin{array}{c}37500 \\
\mathrm{~d}\end{array}$ & $\begin{array}{c}38040 \\
\mathrm{~d}\end{array}$ & $\begin{array}{c}42050 \\
\text { cd }\end{array}$ & $\begin{array}{c}28830 \\
\mathrm{e}\end{array}$ & $\begin{array}{c}9236 \\
\mathrm{f}\end{array}$ & $\begin{array}{c}11560 \\
\mathrm{f}\end{array}$ & $\begin{array}{c}14040 \\
\mathrm{f}\end{array}$ & $\begin{array}{c}10250 \\
\mathrm{f}\end{array}$ \\
\hline LAI & $\begin{array}{c}5.010 \\
\mathrm{~b}\end{array}$ & $\begin{array}{c}5.849 \\
\mathrm{a}\end{array}$ & $\begin{array}{c}4.978 \\
\mathrm{~b}\end{array}$ & $\begin{array}{c}4.527 \\
b c\end{array}$ & $\begin{array}{c}3.750 \\
\mathrm{~d}\end{array}$ & $\begin{array}{c}3.804 \\
\mathrm{~d}\end{array}$ & $\begin{array}{c}4.205 \\
\mathrm{~cd}\end{array}$ & $\begin{array}{c}2.883 \\
\mathrm{e}\end{array}$ & $\begin{array}{c}0.924 \\
\mathrm{f}\end{array}$ & $\begin{array}{c}1.156 \\
\mathrm{f}\end{array}$ & $\begin{array}{c}1.404 \\
\mathrm{f}\end{array}$ & $\begin{array}{c}1.025 \\
\mathrm{f}\end{array}$ \\
\hline $\begin{array}{l}\text { Total plant } \\
\text { dry tan/fed }\end{array}$ & $\begin{array}{c}0.524 \\
b\end{array}$ & $\begin{array}{c}0.624 \\
\mathrm{a}\end{array}$ & $\begin{array}{c}0.721 \\
\mathrm{a}\end{array}$ & \begin{tabular}{|c|}
0.715 \\
$\mathrm{a}$
\end{tabular} & $\begin{array}{c}0.531 \\
\mathrm{~b}\end{array}$ & $\begin{array}{c}0.630 \\
\mathrm{a}\end{array}$ & $\begin{array}{c}0.704 \\
\mathrm{a}\end{array}$ & $\begin{array}{c}0.679 \\
\mathrm{a}\end{array}$ & $\begin{array}{c}0.274 \\
\mathrm{c}\end{array}$ & $\begin{array}{c}0.329 \\
\mathrm{c}\end{array}$ & $\begin{array}{c}0.321 \\
\mathrm{c}\end{array}$ & $\begin{array}{c}0.296 \\
\mathrm{c}\end{array}$ \\
\hline $\begin{array}{l}\text { Leaves dry } \\
\text { weight } \\
\text { (tan/fed) } \\
\end{array}$ & $\begin{array}{c}0.280 \\
\mathrm{c}\end{array}$ & $\begin{array}{c}0.329 \\
\mathrm{~b}\end{array}$ & $\begin{array}{c}0.361 \\
\mathrm{ab}\end{array}$ & $\begin{array}{c}0.365 \\
\mathrm{ab}\end{array}$ & $\begin{array}{c}0.272 \\
\mathrm{c}\end{array}$ & $\begin{array}{c}0.333 \\
a b\end{array}$ & $\begin{array}{c}0.371 \\
\mathrm{a}\end{array}$ & $\begin{array}{c}0.354 \\
a b\end{array}$ & $\begin{array}{c}0.128 \\
\mathrm{e}\end{array}$ & $\begin{array}{c}0.167 \\
\mathrm{~d}\end{array}$ & $\begin{array}{c}0.178 \\
\mathrm{~d}\end{array}$ & $\begin{array}{c}0.128 \\
\mathrm{e}\end{array}$ \\
\hline \begin{tabular}{|l|} 
Stem dry \\
weight \\
(tan/fed) \\
\end{tabular} & $\begin{array}{c}0.244 \\
\mathrm{~d}\end{array}$ & $\begin{array}{c}0.296 \\
\text { bcd }\end{array}$ & $\begin{array}{c}0.361 \\
\mathrm{a}\end{array}$ & $\begin{array}{c}0.347 \\
a b\end{array}$ & $\begin{array}{c}0.259 \\
\mathrm{~d}\end{array}$ & $\begin{array}{c}0.288 \\
\text { cd }\end{array}$ & $\begin{array}{c}0.333 \\
\mathrm{abc}\end{array}$ & $\begin{array}{c}0.325 \\
a b c\end{array}$ & $\begin{array}{c}0.146 \\
\mathrm{e}\end{array}$ & $\begin{array}{c}0.163 \\
\mathrm{e}\end{array}$ & $\begin{array}{c}0.142 \\
\mathrm{e}\end{array}$ & $\begin{array}{c}0.174 \\
\mathrm{e}\end{array}$ \\
\hline $\begin{array}{l}\text { Leaves/stem } \\
\text { ratio }\end{array}$ & $\begin{array}{c}1.087 \\
\mathrm{ab}\end{array}$ & $\begin{array}{c}1.080 \\
\mathrm{ab}\end{array}$ & $\begin{array}{c}0.976 \\
\mathrm{~b}\end{array}$ & \begin{tabular}{|c|}
1.017 \\
$\mathrm{ab}$ \\
\end{tabular} & $\begin{array}{c}1.045 \\
\mathrm{ab}\end{array}$ & $\begin{array}{c}1.139 \\
\mathrm{a}\end{array}$ & $\begin{array}{c}1.106 \\
\mathrm{ab}\end{array}$ & $\begin{array}{c}1.088 \\
a b\end{array}$ & $\begin{array}{c}0.439 \\
\text { de }\end{array}$ & $\begin{array}{c}0.512 \\
\mathrm{~cd}\end{array}$ & $\begin{array}{c}0.630 \\
\mathrm{c}\end{array}$ & \begin{tabular}{|c|}
0.365 \\
$\mathrm{e}$
\end{tabular} \\
\hline \multicolumn{13}{|c|}{$3^{\text {rd }}$ cut } \\
\hline $\begin{array}{l}\text { Plant height } \\
(\mathrm{cm})\end{array}$ & $\begin{array}{c}59.33 \\
\text { bc }\end{array}$ & $\begin{array}{c}58.09 \\
\text { bc }\end{array}$ & $\begin{array}{c}59.84 \\
\text { bc }\end{array}$ & $\begin{array}{c}67.57 \\
\mathrm{a}\end{array}$ & $\begin{array}{c}48.60 \\
\mathrm{~d}\end{array}$ & $\begin{array}{c}55.64 \\
\mathrm{c}\end{array}$ & $\begin{array}{c}62.48 \\
b\end{array}$ & $\begin{array}{c}61.47 \\
b\end{array}$ & $\begin{array}{l}0 \\
\mathrm{e}\end{array}$ & $\begin{array}{l}0 \\
\mathrm{e}\end{array}$ & $\begin{array}{l}0 \\
\mathrm{e}\end{array}$ & $\begin{array}{l}0 \\
\mathrm{e}\end{array}$ \\
\hline $\begin{array}{l}\text { No of tiller / } \\
\mathrm{m}^{2}\end{array}$ & $\begin{array}{c}17.60 \\
\mathrm{a}\end{array}$ & $\begin{array}{c}14.90 \\
\mathrm{c}\end{array}$ & $\begin{array}{c}15.90 \\
\mathrm{ab}\end{array}$ & $\begin{array}{c}17.10 \\
\mathrm{a}\end{array}$ & $\begin{array}{c}12.95 \\
\mathrm{~d}\end{array}$ & $\begin{array}{c}13.40 \\
\mathrm{~cd}\end{array}$ & $\begin{array}{c}13.80 \\
\mathrm{~cd}\end{array}$ & $\begin{array}{c}13.40 \\
\text { cd }\end{array}$ & $\begin{array}{l}0 \\
\mathrm{e}\end{array}$ & $\begin{array}{l}0 \\
\mathrm{e}\end{array}$ & $\begin{array}{l}0 \\
\mathrm{e}\end{array}$ & $\begin{array}{l}0 \\
\mathrm{e}\end{array}$ \\
\hline $\mathrm{LA}\left(\mathrm{cm}^{2} / \mathrm{m}^{2}\right)$ & $\begin{array}{c}5872 \\
\text { abc }\end{array}$ & $\begin{array}{c}6926 \\
\mathrm{a}\end{array}$ & $\begin{array}{c}6673 \\
a b\end{array}$ & $\begin{array}{c}5858 \\
\mathrm{abc}\end{array}$ & $\begin{array}{c}5065 \\
\mathrm{c}\end{array}$ & $\begin{array}{c}5365 \\
\text { bc }\end{array}$ & $\begin{array}{c}6991 \\
\mathrm{c}\end{array}$ & $\begin{array}{c}5841 \\
\mathrm{abc}\end{array}$ & $\begin{array}{l}0 \\
\mathrm{~d}\end{array}$ & $\begin{array}{l}0 \\
\mathrm{~d}\end{array}$ & $\begin{array}{l}0 \\
\mathrm{~d}\end{array}$ & $\begin{array}{l}0 \\
\mathrm{~d}\end{array}$ \\
\hline LAI & $\begin{array}{c}0.587 \\
\mathrm{abc}\end{array}$ & $\begin{array}{c}0.693 \\
\mathrm{a}\end{array}$ & $\begin{array}{c}0.667 \\
a b\end{array}$ & $\begin{array}{c}0.586 \\
\text { abc }\end{array}$ & $\begin{array}{c}0.507 \\
\mathrm{c}\end{array}$ & $\begin{array}{c}0.536 \\
\text { bc }\end{array}$ & $\begin{array}{c}0.699 \\
\mathrm{a}\end{array}$ & $\begin{array}{c}0.584 \\
\text { abc }\end{array}$ & $\begin{array}{l}0 \\
\mathrm{~d}\end{array}$ & $\begin{array}{l}0 \\
\mathrm{~d}\end{array}$ & $\begin{array}{l}0 \\
\mathrm{~d}\end{array}$ & $\begin{array}{l}0 \\
\mathrm{~d}\end{array}$ \\
\hline $\begin{array}{l}\text { Total plant } \\
\text { dry tan/fed }\end{array}$ & $\begin{array}{c}0.190 \\
\mathrm{~b}\end{array}$ & $\begin{array}{c}0.198 \\
\text { b }\end{array}$ & $\begin{array}{c}0.251 \\
\mathrm{a}\end{array}$ & $\begin{array}{c}0.176 \\
\mathrm{c}\end{array}$ & $\begin{array}{c}0.096 \\
\mathrm{~g}\end{array}$ & $\begin{array}{c}0.147 \\
\mathrm{e}\end{array}$ & $\begin{array}{c}0.164 \\
\mathrm{~d}\end{array}$ & $\begin{array}{c}0.112 \\
\mathrm{f}\end{array}$ & $\begin{array}{l}0 \\
\mathrm{~h}\end{array}$ & $\begin{array}{l}0 \\
\mathrm{~h}\end{array}$ & $\begin{array}{l}0 \\
\mathrm{~h}\end{array}$ & $\begin{array}{l}0 \\
\mathrm{~h}\end{array}$ \\
\hline $\begin{array}{l}\text { Leaves dry } \\
\text { weight } \\
\text { (tan/fed) }\end{array}$ & $\begin{array}{c}0.093 \\
\mathrm{~b}\end{array}$ & $\begin{array}{c}0.095 \\
\mathrm{~b}\end{array}$ & $\begin{array}{c}0.117 \\
\mathrm{a}\end{array}$ & \begin{tabular}{|c|}
0.084 \\
bc
\end{tabular} & $\begin{array}{c}0.048 \\
\mathrm{~d}\end{array}$ & $\begin{array}{c}0.073 \\
\mathrm{c}\end{array}$ & $\begin{array}{c}0.078 \\
\mathrm{c}\end{array}$ & $\begin{array}{c}0.052 \\
\mathrm{~d}\end{array}$ & $\begin{array}{l}0 \\
\mathrm{e}\end{array}$ & $\begin{array}{l}0 \\
\mathrm{e}\end{array}$ & $\begin{array}{l}0 \\
\mathrm{e}\end{array}$ & $\begin{array}{l}0 \\
\mathrm{e}\end{array}$ \\
\hline
\end{tabular}

Egypt. J. Agron. 36, No. 1 (2014) 


\begin{tabular}{|c|c|c|c|c|c|c|c|c|c|c|c|c|}
\hline $\begin{array}{l}\text { Stem dry } \\
\text { weight } \\
\text { (tan/fed) }\end{array}$ & $\begin{array}{c}0.097 \\
\text { b }\end{array}$ & $\begin{array}{c}0.097 \\
\mathrm{~b}\end{array}$ & $\begin{array}{c}0.124 \\
\mathrm{a}\end{array}$ & $\begin{array}{c}0.093 \\
\mathrm{~b}\end{array}$ & $\begin{array}{c}0.048 \\
\mathrm{e}\end{array}$ & $\begin{array}{c}0.075 \\
\mathrm{c}\end{array}$ & $\begin{array}{c}0.086 \\
\text { bc }\end{array}$ & $\begin{array}{c}0.060 \\
\mathrm{~d}\end{array}$ & $\begin{array}{l}0 \\
\mathrm{f}\end{array}$ & $\begin{array}{l}0 \\
\mathrm{f}\end{array}$ & $\begin{array}{l}0 \\
\mathrm{f}\end{array}$ & $\begin{array}{l}0 \\
\mathrm{f}\end{array}$ \\
\hline $\begin{array}{l}\text { Leaves/stem } \\
\text { ratio }\end{array}$ & $\begin{array}{c}0.485 \\
\mathrm{ab}\end{array}$ & $\begin{array}{c}0.493 \\
\mathrm{ab}\end{array}$ & $\begin{array}{c}0.479 \\
\mathrm{ab}\end{array}$ & $\begin{array}{c}0.452 \\
\mathrm{ab}\end{array}$ & $\begin{array}{c}0.503 \\
\mathrm{a}\end{array}$ & $\begin{array}{c}0.490 \\
\mathrm{ab}\end{array}$ & $\begin{array}{c}0.454 \\
\mathrm{ab}\end{array}$ & $\begin{array}{c}0.427 \\
\mathrm{~b}\end{array}$ & $\begin{array}{l}0 \\
\mathrm{c}\end{array}$ & $\begin{array}{l}0 \\
\mathrm{c}\end{array}$ & $\begin{array}{l}0 \\
\mathrm{c}\end{array}$ & $\begin{array}{l}0 \\
\mathrm{c}\end{array}$ \\
\hline
\end{tabular}

At the $3^{r d}$ cut, results indicated that using plastic for soil mulching produced the significant of plant height, number of tillers and the leaves/stem ratio at the sowing date $1^{\text {st }}$ May. Whereas, using 2 ton of rice straw for soil mulching had the significant values of the total plant dry weight, leaves dry weight and stem dry weight at the sowing date $1^{\text {st }}$ May. Results showed that there was no re-growth of the peal millet plants at sowing date $1^{\text {st }}$ June. This is may be due to the high temperature which cause high evaporation and led to increase soil salinity.

Results in Table 9 indicated that at the $1^{\text {st }}$ cut using plastic for soil mulching produced the high significant values of the leaves area $/ \mathrm{m}^{2}$, steam dry weight and total plant dry weight at the sowing $1^{\text {st }}$ June under the low saline irrigation water (4500 ppm). Whereas the lowest significant values of the total plant dry weight and steam dry weight were reduced by using 2 ton of rice straw for soil mulching at $1^{\text {st }}$ June under the high saline irrigation water ( $\left.8000 \mathrm{ppm}\right)$.

At the $2^{\text {nd }}$ cut using 2 ton ton of rice straw for soil mulching produced the high significant values of the total plant dry weight at the sowing $1^{\text {st }}$ and $2^{\text {nd }}$ sowing dates followed by using plastic for soil mulching under the low saline irrigation water (4500 ppm). This is may be due to the adequate amount of the rice straw for keeping the soil surface against the evaporation and save soil moisture. Whereas the lowest significant values of the total plant dry weight and steam dry weight were reduced by using without soil mulching at $1^{\text {st }}$ May and $15^{\text {th }}$ May under the high saline irrigation water $(8000 \mathrm{ppm})$.

At the $3^{\text {rd }}$ cut results indicated that using 2 ton of rice straw for soil mulching produced the high significant values of the total plant dry weight, steam dry weight and leaves dry weight at the sowing $1^{s t}$ sowing date under the low saline irrigation water $(4500 \mathrm{ppm})$. Whereas, there was no re-growth of pearl millet at the $1^{\text {st }}$ June sowing date under the low saline irrigation water $(4500 \mathrm{ppm})$ and at the three sowing dates under the high saline irrigation water $(8000 \mathrm{ppm})$. 
TABLE 9. Effect of the interaction between saline water irrigation levels, sowing dates and soil mulching on some growth characters and forage yield of pearl millet (average the two seasons 2011 and 2012).

\begin{tabular}{|c|c|c|c|c|c|c|c|c|c|c|c|c|}
\hline \multicolumn{7}{|c|}{ Plant height $(\mathrm{cm})$} & \multicolumn{6}{|c|}{ No. of tiller $/ \mathrm{m}^{2}$} \\
\hline & \multicolumn{3}{|c|}{ 4500ppm } & \multicolumn{3}{|c|}{ 8000ppm } & \multicolumn{3}{|c|}{ 4500ppm } & \multicolumn{3}{|c|}{ 8000ppm } \\
\hline date & $1 / 5$ & $15 / 5$ & $1 / 6$ & $1 / 5$ & $15 / 5$ & $1 / 6$ & $1 / 5$ & $15 / 5$ & $1 / 6$ & $1 / 5$ & $15 / 5$ & $1 / 6$ \\
\hline $\begin{array}{c}\text { Soil } \\
\text { mulching }\end{array}$ & \multicolumn{12}{|c|}{$1^{s t} \mathrm{cut}$} \\
\hline Without & $\begin{array}{c}91.63 \\
\text { def }\end{array}$ & $\begin{array}{c}97.58 \\
\text { bcd }\end{array}$ & $\begin{array}{c}108.1 \\
\mathrm{a} \\
\end{array}$ & $\begin{array}{c}78.67 \\
\mathrm{~g} \\
\end{array}$ & $\begin{array}{c}93.37 \\
\text { def }\end{array}$ & $\begin{array}{c}89.75 \\
\text { ef }\end{array}$ & $\begin{array}{c}147.8 \\
\mathrm{a} \\
\end{array}$ & $\begin{array}{c}118.5 \\
\text { de }\end{array}$ & $\begin{array}{c}99.4 \\
\mathrm{f} \\
\end{array}$ & $\begin{array}{c}104.2 \\
\mathrm{f}\end{array}$ & $\begin{array}{c}66.40 \\
\text { hij } \\
\end{array}$ & $\begin{array}{c}45.00 \\
\mathrm{k}\end{array}$ \\
\hline $\begin{array}{l}1 \text { ton/fed } \\
\text { straw }\end{array}$ & $\begin{array}{l}97.9 \\
\text { bcd }\end{array}$ & $\begin{array}{c}94.80 \\
\text { cde }\end{array}$ & $\begin{array}{c}101.1 \\
\mathrm{abc}\end{array}$ & $\begin{array}{c}91.94 \\
\text { def }\end{array}$ & $\begin{array}{c}90.55 \\
\text { def }\end{array}$ & $\begin{array}{c}93.12 \\
\text { def }\end{array}$ & $\begin{array}{c}121.1 \\
\mathrm{~cd}\end{array}$ & $\begin{array}{c}99.9 \\
\mathrm{f}\end{array}$ & $\begin{array}{c}131.6 \\
\text { bc }\end{array}$ & $\begin{array}{c}69.00 \\
\text { hi }\end{array}$ & $\begin{array}{c}69.20 \\
\text { hi }\end{array}$ & $\begin{array}{c}69.40 \\
\text { hi }\end{array}$ \\
\hline $\begin{array}{l}2 \text { ton/fed } \\
\text { straw }\end{array}$ & $\begin{array}{c}96.84 \\
\text { b-e }\end{array}$ & $\begin{array}{c}93.98 \\
\text { c-f }\end{array}$ & $\begin{array}{c}103.1 \\
\mathrm{ab}\end{array}$ & $\begin{array}{c}92.33 \\
\text { def }\end{array}$ & $\begin{array}{c}91.87 \\
\text { def }\end{array}$ & $\begin{array}{c}86.88 \\
\mathrm{f}\end{array}$ & $\begin{array}{c}131.1 \\
\text { bc }\end{array}$ & $\begin{array}{c}98.30 \\
\mathrm{f}\end{array}$ & $\begin{array}{c}109.8 \\
\text { ef }\end{array}$ & $\begin{array}{c}78.00 \\
\text { gh }\end{array}$ & $\begin{array}{c}61.80 \\
\mathrm{ij}\end{array}$ & $\begin{array}{c}58.40 \\
\mathrm{ij} \\
\end{array}$ \\
\hline Plastic & $\begin{array}{c}96.5 \\
\text { b-e }\end{array}$ & $\begin{array}{c}105.9 \\
\mathrm{a}\end{array}$ & $\begin{array}{c}105.8 \\
\mathrm{a}\end{array}$ & $\begin{array}{c}91.62 \\
\text { def }\end{array}$ & $\begin{array}{c}95.87 \\
\text { b-e }\end{array}$ & $\begin{array}{c}94.10 \\
\mathrm{c}-\mathrm{f}\end{array}$ & $\begin{array}{c}103.3 \\
\mathrm{f}\end{array}$ & $\begin{array}{c}134.7 \\
\mathrm{~b}\end{array}$ & $\begin{array}{c}141.5 \\
\mathrm{ab}\end{array}$ & $\begin{array}{c}87.40 \\
\mathrm{~g}\end{array}$ & $\begin{array}{c}56.80 \\
\mathrm{j}\end{array}$ & $\begin{array}{c}44.00 \\
\mathrm{k}\end{array}$ \\
\hline \multicolumn{13}{|c|}{$2^{\text {nd }}$ cut } \\
\hline Without & $\begin{array}{c}116.7 \\
\text { cde }\end{array}$ & $\begin{array}{c}115.3 \\
\text { de }\end{array}$ & $\begin{array}{c}117.2 \\
\text { cde }\end{array}$ & $\begin{array}{c}90.09 \\
\mathrm{i} \\
\end{array}$ & $\begin{array}{c}94.57 \\
\mathrm{i}\end{array}$ & $\begin{array}{l}0 \\
\mathrm{~J}\end{array}$ & $\begin{array}{c}96.10 \\
\text { cde }\end{array}$ & $\begin{array}{c}92.80 \\
\text { cde }\end{array}$ & $\begin{array}{c}51.60 \\
\mathrm{~g}\end{array}$ & $\begin{array}{c}43.40 \\
\text { ghi }\end{array}$ & $\begin{array}{c}44.10 \\
\text { gh }\end{array}$ & $\begin{array}{l}0 \\
\mathrm{k} \\
\end{array}$ \\
\hline $\begin{array}{l}\text { 1ton/fed } \\
\text { straw }\end{array}$ & $\begin{array}{c}117.0 \\
\text { cde }\end{array}$ & $\begin{array}{c}108.7 \\
\mathrm{fg}\end{array}$ & $\begin{array}{c}108.7 \\
\mathrm{fg}\end{array}$ & $\begin{array}{c}95.75 \\
\mathrm{i} \\
\end{array}$ & $\begin{array}{c}92.72 \\
\mathrm{i}\end{array}$ & $\begin{array}{l}0 \\
\mathrm{~J} \\
\end{array}$ & $\begin{array}{c}110.5 \\
\mathrm{ab}\end{array}$ & $\begin{array}{c}104.5 \\
\text { bc }\end{array}$ & $\begin{array}{c}89.40 \\
\text { de }\end{array}$ & $\begin{array}{c}38.30 \\
\text { hi }\end{array}$ & $\begin{array}{c}37.00 \\
\text { hij }\end{array}$ & $\begin{array}{l}0 \\
\mathrm{k}\end{array}$ \\
\hline $\begin{array}{l}2 \text { ton/fed } \\
\text { straw }\end{array}$ & $\begin{array}{c}128.0 \\
\mathrm{a}\end{array}$ & $\begin{array}{c}122.6 \\
\text { abc }\end{array}$ & $\begin{array}{c}111.7 \\
\text { ef }\end{array}$ & $\begin{array}{c}106.5 \\
\mathrm{fg}\end{array}$ & $\begin{array}{c}95.73 \\
\mathrm{i}\end{array}$ & $\begin{array}{l}0 \\
\mathrm{~J}\end{array}$ & $\begin{array}{c}118.9 \\
\mathrm{a}\end{array}$ & $\begin{array}{c}91.80 \\
\text { cde }\end{array}$ & $\begin{array}{c}74.20 \\
\mathrm{f}\end{array}$ & $\begin{array}{c}32.00 \\
\mathrm{ij}\end{array}$ & $\begin{array}{c}48.00 \\
\mathrm{gh}\end{array}$ & $\begin{array}{l}0 \\
\mathrm{k}\end{array}$ \\
\hline Plastic & $\begin{array}{c}128.0 \\
\mathrm{a}\end{array}$ & $\begin{array}{c}124.4 \\
\mathrm{ab}\end{array}$ & $\begin{array}{c}119.5 \\
\text { bcd }\end{array}$ & $\begin{array}{c}102.4 \\
\mathrm{gh}\end{array}$ & $\begin{array}{c}96.77 \\
\text { hi }\end{array}$ & $\begin{array}{l}0 \\
\mathrm{~J}\end{array}$ & $\begin{array}{c}101.6 \\
\text { bcd }\end{array}$ & $\begin{array}{c}85.50 \\
\text { ef }\end{array}$ & $\begin{array}{c}84.60 \\
\text { ef }\end{array}$ & $\begin{array}{c}25.80 \\
\mathrm{j}\end{array}$ & $\begin{array}{c}56.20 \\
\mathrm{~g}\end{array}$ & $\begin{array}{l}0 \\
\mathrm{k}\end{array}$ \\
\hline \multicolumn{13}{|c|}{$3^{\text {rd }}$ cut } \\
\hline Without & $\begin{array}{c}118.7 \\
\mathrm{bc}\end{array}$ & $\begin{array}{c}97.20 \\
\mathrm{e}\end{array}$ & $\begin{array}{r}0 \\
\mathrm{f}\end{array}$ & $\begin{array}{l}0 \\
\mathrm{f}\end{array}$ & $\begin{array}{l}0 \\
\mathrm{f}\end{array}$ & $\begin{array}{l}0 \\
\mathrm{f}\end{array}$ & $\begin{array}{c}35.20 \\
\mathrm{a} \\
\end{array}$ & $\begin{array}{c}25.90 \\
\mathrm{~d} \\
\end{array}$ & $\begin{array}{l}0 \\
\mathrm{e}\end{array}$ & $\begin{array}{l}0 \\
\mathrm{e}\end{array}$ & $\begin{array}{l}0 \\
\mathrm{e}\end{array}$ & $\begin{array}{l}0 \\
\mathrm{e}\end{array}$ \\
\hline $\begin{array}{l}\text { 1ton/fed } \\
\text { straw }\end{array}$ & $\begin{array}{c}116.2 \\
\mathrm{~cd}\end{array}$ & $\begin{array}{c}111.3 \\
\mathrm{~d}\end{array}$ & $\begin{array}{l}0 \\
\mathrm{f}\end{array}$ & $\begin{array}{l}0 \\
\mathrm{f}\end{array}$ & $\begin{array}{l}0 \\
\mathrm{f}\end{array}$ & $\begin{array}{l}0 \\
\mathrm{f}\end{array}$ & $\begin{array}{c}29.80 \\
\text { bc }\end{array}$ & $\begin{array}{c}26.80 \\
\mathrm{~d}\end{array}$ & $\begin{array}{l}0 \\
\mathrm{e}\end{array}$ & $\begin{array}{l}0 \\
\mathrm{e}\end{array}$ & $\begin{array}{l}0 \\
\mathrm{e}\end{array}$ & $\begin{array}{l}0 \\
\mathrm{e}\end{array}$ \\
\hline $\begin{array}{l}2 \text { ton/fed } \\
\text { straw }\end{array}$ & $\begin{array}{c}119.7 \\
\mathrm{bc}\end{array}$ & $\begin{array}{c}125.0 \\
\mathrm{~b}\end{array}$ & $\begin{array}{l}0 \\
\mathrm{f}\end{array}$ & $\begin{array}{l}0 \\
\mathrm{f}\end{array}$ & $\begin{array}{l}0 \\
\mathrm{f}\end{array}$ & $\begin{array}{l}0 \\
\mathrm{f}\end{array}$ & $\begin{array}{c}31.80 \\
\mathrm{~b}\end{array}$ & $\begin{array}{c}27.60 \\
\mathrm{~cd}\end{array}$ & $\begin{array}{l}0 \\
\mathrm{e}\end{array}$ & $\begin{array}{l}0 \\
\mathrm{e}\end{array}$ & $\begin{array}{l}0 \\
\mathrm{e}\end{array}$ & $\begin{array}{l}0 \\
\mathrm{e} \\
\end{array}$ \\
\hline Plastic & $\begin{array}{c}135.1 \\
\mathrm{a}\end{array}$ & $\begin{array}{c}122.9 \\
\mathrm{~b}\end{array}$ & $\begin{array}{l}0 \\
\mathrm{f}\end{array}$ & $\begin{array}{l}0 \\
\mathrm{f}\end{array}$ & $\begin{array}{l}0 \\
\mathrm{f}\end{array}$ & $\begin{array}{l}0 \\
\mathrm{f}\end{array}$ & $\begin{array}{c}34.20 \\
\mathrm{a}\end{array}$ & $\begin{array}{c}26.80 \\
\mathrm{~d}\end{array}$ & $\begin{array}{l}0 \\
\mathrm{e}\end{array}$ & $\begin{array}{l}0 \\
\mathrm{e}\end{array}$ & $\begin{array}{l}0 \\
\mathrm{e}\end{array}$ & $\begin{array}{l}0 \\
\mathrm{e}\end{array}$ \\
\hline
\end{tabular}

Egypt. J. Agron. 36, No. 1 (2014) 
TABLE 9. Cont.

\begin{tabular}{|c|c|c|c|c|c|c|c|c|c|c|c|c|}
\hline \multicolumn{7}{|c|}{$\mathbf{L A}\left(\mathrm{cm}^{2} / \mathrm{m}^{2}\right)$} & \multicolumn{6}{|c|}{ LAI } \\
\hline & \multicolumn{3}{|c|}{$4500 p p m$} & \multicolumn{3}{|c|}{$8000 p p m$} & \multicolumn{3}{|c|}{$4500 \mathrm{ppm}$} & \multicolumn{3}{|c|}{$8000 p p m$} \\
\hline $\begin{array}{l}\text { Planting } \\
\text { date }\end{array}$ & $1 / 5$ & $15 / 5$ & $1 / 6$ & $1 / 5$ & $15 / 5$ & $1 / 6$ & $1 / 5$ & $15 / 5$ & $1 / 6$ & $1 / 5$ & $15 / 5$ & $1 / 6$ \\
\hline $\begin{array}{l}\text { Soil } \\
\text { mulching }\end{array}$ & \multicolumn{12}{|c|}{$1^{s t}$ cut } \\
\hline Without & $\begin{array}{c}77920 \\
\text { bc }\end{array}$ & $\begin{array}{c}67850 \\
\mathrm{~cd}\end{array}$ & $\begin{array}{c}77250 \\
b c\end{array}$ & $\begin{array}{c}16880 \\
\mathrm{e}\end{array}$ & $\begin{array}{c}12000 \\
\mathrm{e}\end{array}$ & $\begin{array}{c}10900 \\
\mathrm{e}\end{array}$ & $\begin{array}{c}7.792 \\
\text { bc }\end{array}$ & $\begin{array}{c}6.785 \\
\mathrm{~cd}\end{array}$ & $\begin{array}{c}7.725 \\
\text { bc }\end{array}$ & $\begin{array}{c}1.688 \\
\mathrm{e}\end{array}$ & $\begin{array}{c}1.200 \\
\mathrm{e}\end{array}$ & $\begin{array}{c}1.090 \\
\mathrm{e}\end{array}$ \\
\hline $\begin{array}{l}1 \text { ton/fed } \\
\text { straw }\end{array}$ & $\begin{array}{c}76430 \\
\text { bc }\end{array}$ & $\begin{array}{c}65350 \\
\mathrm{~cd}\end{array}$ & $\begin{array}{c}98540 \\
\mathrm{a}\end{array}$ & $\begin{array}{c}15620 \\
\mathrm{e}\end{array}$ & $\begin{array}{c}10090 \\
\mathrm{e}\end{array}$ & $\begin{array}{c}11110 \\
\mathrm{e}\end{array}$ & $\begin{array}{c}7.643 \\
b c\end{array}$ & $\begin{array}{c}6.535 \\
\mathrm{~cd}\end{array}$ & $\begin{array}{c}9.854 \\
\mathrm{a}\end{array}$ & $\begin{array}{c}1.562 \\
\mathrm{e}\end{array}$ & $\begin{array}{c}1.009 \\
\mathrm{e}\end{array}$ & $\begin{array}{c}1.111 \\
\mathrm{e}\end{array}$ \\
\hline $\begin{array}{l}2 \text { ton/fed } \\
\text { straw }\end{array}$ & $\begin{array}{c}83990 \\
\text { b }\end{array}$ & $\begin{array}{c}60480 \\
\text { d }\end{array}$ & $\begin{array}{c}68200 \\
\mathrm{~cd}\end{array}$ & $\begin{array}{c}14900 \\
\mathrm{e}\end{array}$ & $\begin{array}{c}12230 \\
\mathrm{e}\end{array}$ & $\begin{array}{c}13620 \\
\mathrm{e}\end{array}$ & $\begin{array}{c}8.399 \\
\text { b }\end{array}$ & $\begin{array}{c}6.048 \\
\mathrm{~d}\end{array}$ & $\begin{array}{c}6.820 \\
\mathrm{~cd}\end{array}$ & $\begin{array}{c}1.490 \\
\mathrm{e}\end{array}$ & $\begin{array}{c}1.223 \\
\mathrm{e}\end{array}$ & $\begin{array}{c}1.362 \\
\mathrm{e}\end{array}$ \\
\hline Plastic & $\begin{array}{c}78180 \\
\text { bc }\end{array}$ & $\begin{array}{c}73060 \\
\text { bcd }\end{array}$ & $\begin{array}{c}104100 \\
\mathrm{a}\end{array}$ & $\begin{array}{c}13830 \\
\mathrm{e}\end{array}$ & $\begin{array}{c}11170 \\
\mathrm{e}\end{array}$ & $\begin{array}{c}10270 \\
\mathrm{e}\end{array}$ & $\begin{array}{c}7.818 \\
\text { bc }\end{array}$ & $\begin{array}{c}7.306 \\
\text { bcd }\end{array}$ & $\begin{array}{c}10.41 \\
\mathrm{a}\end{array}$ & $\begin{array}{c}1.383 \\
\mathrm{e}\end{array}$ & $\begin{array}{c}1.117 \\
\mathrm{e}\end{array}$ & $\begin{array}{c}1.027 \\
\mathrm{e}\end{array}$ \\
\hline \multicolumn{13}{|c|}{$2^{\text {nd }}$ cut } \\
\hline Without & $\begin{array}{c}93140 \\
\text { b }\end{array}$ & $\begin{array}{c}70780 \\
\mathrm{~d}\end{array}$ & $\begin{array}{c}18470 \\
\mathrm{~g}\end{array}$ & $\begin{array}{c}7065 \\
\mathrm{~h}\end{array}$ & $\begin{array}{c}4222 \\
\mathrm{~h}\end{array}$ & $\begin{array}{l}0 \\
\mathrm{~h}\end{array}$ & $\begin{array}{c}9.314 \\
b\end{array}$ & $\begin{array}{c}7.078 \\
\mathrm{~d}\end{array}$ & $\begin{array}{c}1.847 \\
\mathrm{~g}\end{array}$ & $\begin{array}{c}0.707 \\
\mathrm{~h}\end{array}$ & $\begin{array}{c}0.422 \\
\mathrm{~h}\end{array}$ & $\begin{array}{l}0 \\
\mathrm{~h}\end{array}$ \\
\hline $\begin{array}{l}1 \text { ton/fed } \\
\text { straw }\end{array}$ & $\begin{array}{c}109200 \\
\mathrm{a}\end{array}$ & $\begin{array}{c}69980 \\
\mathrm{~d}\end{array}$ & $\begin{array}{c}23110 \\
\mathrm{fg}\end{array}$ & $\begin{array}{c}7772 \\
\text { h }\end{array}$ & $\begin{array}{c}6098 \\
\mathrm{~h}\end{array}$ & $\begin{array}{l}0 \\
\mathrm{~h}\end{array}$ & $\begin{array}{c}10.92 \\
\mathrm{a}\end{array}$ & $\begin{array}{c}6.998 \\
\mathrm{~d}\end{array}$ & $\begin{array}{c}2.311 \\
\mathrm{fg}\end{array}$ & $\begin{array}{c}0.777 \\
\mathrm{~h}\end{array}$ & $\begin{array}{c}0.610 \\
\mathrm{~h}\end{array}$ & $\begin{array}{l}0 \\
\mathrm{~h}\end{array}$ \\
\hline $\begin{array}{l}2 \text { ton/fed } \\
\text { straw }\end{array}$ & $\begin{array}{c}90260 \\
\text { b }\end{array}$ & $\begin{array}{c}78370 \\
\mathrm{~cd}\end{array}$ & $\begin{array}{c}28070 \\
\mathrm{f}\end{array}$ & $\begin{array}{c}9299 \\
\mathrm{~h}\end{array}$ & $\begin{array}{c}5735 \\
\mathrm{~h}\end{array}$ & $\begin{array}{l}0 \\
\mathrm{~h}\end{array}$ & $\begin{array}{c}9.026 \\
b\end{array}$ & $\begin{array}{c}7.837 \\
\mathrm{~cd}\end{array}$ & $\begin{array}{c}2.807 \\
\mathrm{f}\end{array}$ & $\begin{array}{c}0.930 \\
\mathrm{~h}\end{array}$ & $\begin{array}{c}0.574 \\
\mathrm{~h}\end{array}$ & $\begin{array}{l}0 \\
\mathrm{~h}\end{array}$ \\
\hline Plastic & $\begin{array}{c}85850 \\
\text { bc }\end{array}$ & $\begin{array}{c}53030 \\
\mathrm{e}\end{array}$ & $\begin{array}{c}20500 \\
\mathrm{fg}\end{array}$ & $\begin{array}{c}4693 \\
\text { h }\end{array}$ & $\begin{array}{c}4637 \\
\mathrm{~h}\end{array}$ & $\begin{array}{l}0 \\
\mathrm{~h}\end{array}$ & $\begin{array}{c}8.585 \\
\text { bc }\end{array}$ & $\begin{array}{c}5.303 \\
\mathrm{e}\end{array}$ & $\begin{array}{c}2.050 \\
\mathrm{fg}\end{array}$ & $\begin{array}{c}0.4670 \\
\mathrm{~h}\end{array}$ & $\begin{array}{c}0.464 \\
\mathrm{~h}\end{array}$ & $\begin{array}{l}0 \\
\mathrm{~h}\end{array}$ \\
\hline & & & & & & $3^{r d} \mathrm{cut}$ & & & & & & \\
\hline Without & $\begin{array}{c}11740 \\
\text { bc }\end{array}$ & $\begin{array}{c}10130 \\
\mathrm{c}\end{array}$ & $\begin{array}{l}0 \\
d\end{array}$ & $\begin{array}{l}0 \\
\mathrm{~d}\end{array}$ & $\begin{array}{l}0 \\
d\end{array}$ & $\begin{array}{l}0 \\
\mathrm{~d}\end{array}$ & $\begin{array}{c}1.174 \\
\text { bc }\end{array}$ & $\begin{array}{c}1.013 \\
\mathrm{c}\end{array}$ & $\begin{array}{l}0 \\
\mathrm{~d}\end{array}$ & $\begin{array}{l}0 \\
\mathrm{~d}\end{array}$ & $\begin{array}{l}0 \\
\mathrm{~d}\end{array}$ & $\begin{array}{l}0 \\
\mathrm{~d}\end{array}$ \\
\hline $\begin{array}{l}1 \text { ton/fed } \\
\text { straw }\end{array}$ & $\begin{array}{c}13850 \\
\mathrm{a}\end{array}$ & $\begin{array}{c}10730 \\
\mathrm{c}\end{array}$ & $\begin{array}{l}0 \\
d\end{array}$ & $\begin{array}{l}0 \\
\mathrm{~d}\end{array}$ & $\begin{array}{l}0 \\
\mathrm{~d}\end{array}$ & $\begin{array}{l}0 \\
\mathrm{~d}\end{array}$ & $\begin{array}{c}1.385 \\
\mathrm{a}\end{array}$ & $\begin{array}{c}1.073 \\
\mathrm{c}\end{array}$ & $\begin{array}{l}0 \\
\mathrm{~d}\end{array}$ & $\begin{array}{l}0 \\
\mathrm{~d}\end{array}$ & $\begin{array}{l}0 \\
\mathrm{~d}\end{array}$ & $\begin{array}{l}0 \\
\mathrm{~d}\end{array}$ \\
\hline $\begin{array}{l}2 \text { ton/fed } \\
\text { straw }\end{array}$ & $\begin{array}{c}13350 \\
\mathrm{ab}\end{array}$ & $\begin{array}{c}13980 \\
\mathrm{a}\end{array}$ & $\begin{array}{l}0 \\
d\end{array}$ & $\begin{array}{l}0 \\
\mathrm{~d}\end{array}$ & $\begin{array}{l}0 \\
d\end{array}$ & $\begin{array}{l}0 \\
\mathrm{~d}\end{array}$ & $\begin{array}{c}1.335 \\
\mathrm{ab}\end{array}$ & $\begin{array}{c}1.398 \\
\mathrm{a}\end{array}$ & $\begin{array}{l}0 \\
\mathrm{~d}\end{array}$ & $\begin{array}{l}0 \\
\mathrm{~d}\end{array}$ & $\begin{array}{l}0 \\
\mathrm{~d}\end{array}$ & $\begin{array}{l}0 \\
\mathrm{~d}\end{array}$ \\
\hline Plastic & $\begin{array}{c}11720 \\
\text { bc }\end{array}$ & $\begin{array}{c}11680 \\
\text { bc }\end{array}$ & $\begin{array}{l}0 \\
\mathrm{~d}\end{array}$ & $\begin{array}{l}0 \\
\mathrm{~d}\end{array}$ & $\begin{array}{l}0 \\
\mathrm{~d}\end{array}$ & $\begin{array}{l}0 \\
\mathrm{~d}\end{array}$ & $\begin{array}{c}1.172 \\
\text { bc }\end{array}$ & $\begin{array}{c}1.168 \\
\text { bc }\end{array}$ & $\begin{array}{l}0 \\
\mathrm{~d}\end{array}$ & $\begin{array}{l}0 \\
\mathrm{~d}\end{array}$ & $\begin{array}{l}0 \\
\mathrm{~d}\end{array}$ & $\begin{array}{l}0 \\
\mathrm{~d}\end{array}$ \\
\hline
\end{tabular}


TABLE 9. Cont.

\begin{tabular}{|c|c|c|c|c|c|c|c|c|c|c|c|c|}
\hline \multicolumn{7}{|c|}{ Dry wt } & \multicolumn{6}{|c|}{ Dry wt leave } \\
\hline & \multicolumn{3}{|c|}{ 4500ppm } & \multicolumn{3}{|c|}{ 8000ppm } & \multicolumn{3}{|c|}{ 4500ppm } & \multicolumn{3}{|c|}{ 8000ppm } \\
\hline $\begin{array}{l}\text { Planting } \\
\text { date }\end{array}$ & $1 / 5$ & $15 / 5$ & $1 / 6$ & $1 / 5$ & $15 / 5$ & $1 / 6$ & $1 / 5$ & $15 / 5$ & $1 / 6$ & $1 / 5$ & $15 / 5$ & $1 / 6$ \\
\hline $\begin{array}{l}\text { Soil } \\
\text { mulching }\end{array}$ & \multicolumn{12}{|c|}{$1^{s t} \mathrm{cut}$} \\
\hline Without & $\begin{array}{c}0.748 \\
\text { b-f }\end{array}$ & $\begin{array}{c}0.804 \\
\text { b-e }\end{array}$ & $\begin{array}{c}0.849 \\
\text { a-d }\end{array}$ & $\begin{array}{c}0.441 \\
\mathrm{jk}\end{array}$ & $\begin{array}{c}0.511 \\
\mathrm{~g}-\mathrm{k}\end{array}$ & $\begin{array}{c}0.467 \\
\mathrm{ijk}\end{array}$ & $\begin{array}{c}0.480 \\
\text { abc }\end{array}$ & $\begin{array}{c}0.503 \\
\text { abc }\end{array}$ & $\begin{array}{c}0.464 \\
\text { abc }\end{array}$ & $\begin{array}{c}0.245 \\
\text { fg }\end{array}$ & $\begin{array}{c}0.284 \\
\text { efg }\end{array}$ & $\begin{array}{c}0.221 \\
\mathrm{fg}\end{array}$ \\
\hline $\begin{array}{l}\text { 1ton/fed } \\
\text { straw }\end{array}$ & $\begin{array}{c}0.648 \\
c-j\end{array}$ & $\begin{array}{c}0.695 \\
c-i\end{array}$ & $\begin{array}{c}0.860 \\
\text { abc }\end{array}$ & $\begin{array}{c}0.466 \\
\mathrm{ijk}\end{array}$ & $\begin{array}{c}0.503 \\
\text { h-k }\end{array}$ & $\begin{array}{c}0.493 \\
\text { h-k }\end{array}$ & $\begin{array}{c}0.390 \\
\text { cde }\end{array}$ & $\begin{array}{c}0.434 \\
\text { bcd }\end{array}$ & $\begin{array}{c}0.517 \\
a b\end{array}$ & $\begin{array}{c}0.242 \\
\mathrm{fg}\end{array}$ & $\begin{array}{c}0.289 \\
\text { efg }\end{array}$ & $\begin{array}{c}0.238 \\
\mathrm{fg}\end{array}$ \\
\hline $\begin{array}{l}2 \text { ton/fed } \\
\text { straw }\end{array}$ & $\begin{array}{c}0.703 \\
\text { c-h }\end{array}$ & $\begin{array}{c}0.630 \\
\text { d-k }\end{array}$ & $\begin{array}{c}0.738 \\
\text { b-g }\end{array}$ & $\begin{array}{c}0.473 \\
\text { h-k }\end{array}$ & $\begin{array}{c}0.531 \\
\mathrm{f}-\mathrm{k}\end{array}$ & $\begin{array}{c}0.399 \\
\mathrm{k}\end{array}$ & $\begin{array}{c}0.456 \\
\text { a-d }\end{array}$ & $\begin{array}{c}0.383 \\
\text { cde }\end{array}$ & $\begin{array}{c}0.435 \\
\text { bcd }\end{array}$ & $\begin{array}{c}0.262 \\
\mathrm{fg}\end{array}$ & $\begin{array}{c}0.306 \\
\text { efg }\end{array}$ & $\begin{array}{c}0.200 \\
\mathrm{~g}\end{array}$ \\
\hline Plastic & $\begin{array}{c}0.801 \\
\text { b-e }\end{array}$ & $\begin{array}{c}0.945 \\
\mathrm{ab}\end{array}$ & $\begin{array}{c}1.025 \\
\mathrm{a}\end{array}$ & $\begin{array}{c}0.538 \\
\mathrm{f}-\mathrm{k}\end{array}$ & $\begin{array}{c}0.595 \\
\text { e-k }\end{array}$ & $\begin{array}{c}0.455 \\
\mathrm{jk}\end{array}$ & $\begin{array}{c}0.494 \\
\text { abc }\end{array}$ & $\begin{array}{c}0.568 \\
\mathrm{a}\end{array}$ & $\begin{array}{c}0.569 \\
\mathrm{a}\end{array}$ & $\begin{array}{c}0.293 \\
\text { efg }\end{array}$ & $\begin{array}{c}0.340 \\
\text { def }\end{array}$ & $\begin{array}{c}0.200 \\
\mathrm{~g}\end{array}$ \\
\hline \multicolumn{13}{|c|}{$2^{\text {nd }}$ cut } \\
\hline Without & $\begin{array}{c}0.728 \\
\text { bc }\end{array}$ & $\begin{array}{c}0.714 \\
\text { bc }\end{array}$ & $\begin{array}{c}0.547 \\
\text { d-g }\end{array}$ & $\begin{array}{c}0.320 \\
\mathrm{~h}\end{array}$ & $\begin{array}{c}0.348 \\
\mathrm{~h}\end{array}$ & $\begin{array}{l}0 \\
\mathrm{i}\end{array}$ & $\begin{array}{c}0.414 \\
\mathrm{~cd}\end{array}$ & $\begin{array}{c}0.373 \\
\text { de }\end{array}$ & $\begin{array}{c}0.256 \\
\mathrm{fg}\end{array}$ & $\begin{array}{c}0.146 \\
\mathrm{i}\end{array}$ & $\begin{array}{c}0.172 \\
\text { hi }\end{array}$ & $\begin{array}{l}0 \\
\mathrm{j}\end{array}$ \\
\hline $\begin{array}{l}1 \text { ton/fed } \\
\text { straw }\end{array}$ & $\begin{array}{c}0.820 \\
\mathrm{ab} \\
\end{array}$ & $\begin{array}{c}0.842 \\
\mathrm{ab} \\
\end{array}$ & $\begin{array}{c}0.658 \\
\mathrm{~cd} \\
\end{array}$ & $\begin{array}{c}0.429 \\
\mathrm{gh} \\
\end{array}$ & $\begin{array}{c}0.417 \\
\text { gh }\end{array}$ & $\begin{array}{l}0 \\
\mathrm{i} \\
\end{array}$ & $\begin{array}{c}0.450 \\
\mathrm{bc}\end{array}$ & $\begin{array}{c}0.455 \\
\mathrm{bc}\end{array}$ & $\begin{array}{c}0.333 \\
\mathrm{e} \\
\end{array}$ & $\begin{array}{c}0.208 \\
\text { fgh } \\
\end{array}$ & $\begin{array}{c}0.211 \\
\text { fgh }\end{array}$ & $\begin{array}{l}0 \\
\mathrm{j}\end{array}$ \\
\hline $\begin{array}{l}\text { 2ton/fed } \\
\text { straw }\end{array}$ & $\begin{array}{c}0.940 \\
\mathrm{a}\end{array}$ & $\begin{array}{c}0.915 \\
\mathrm{a}\end{array}$ & $\begin{array}{c}0.641 \\
\text { cde }\end{array}$ & $\begin{array}{c}0.503 \\
\text { efg }\end{array}$ & $\begin{array}{c}0.493 \\
\mathrm{fg}\end{array}$ & $\begin{array}{l}0 \\
\mathrm{i}\end{array}$ & $\begin{array}{c}0.524 \\
\mathrm{a}\end{array}$ & $\begin{array}{c}0.494 \\
\mathrm{ab}\end{array}$ & $\begin{array}{c}0.357 \\
\mathrm{e}\end{array}$ & $\begin{array}{c}0.197 \\
\text { ghi }\end{array}$ & $\begin{array}{c}0.248 \\
\mathrm{fg}\end{array}$ & $\begin{array}{l}0 \\
\mathrm{j}\end{array}$ \\
\hline Plastic & $\begin{array}{c}0.905 \\
\mathrm{a}\end{array}$ & $\begin{array}{c}0.819 \\
\mathrm{ab}\end{array}$ & $\begin{array}{c}0.591 \\
\text { d-f }\end{array}$ & $\begin{array}{c}0.525 \\
\mathrm{~d}-\mathrm{g} \\
\end{array}$ & $\begin{array}{c}0.539 \\
\text { d-g }\end{array}$ & $\begin{array}{l}0 \\
\mathrm{i}\end{array}$ & $\begin{array}{c}0.515 \\
\mathrm{a} \\
\end{array}$ & $\begin{array}{c}0.445 \\
\mathrm{bc}\end{array}$ & $\begin{array}{c}0.256 \\
\mathrm{fg}\end{array}$ & $\begin{array}{c}0.216 \\
\text { fgh }\end{array}$ & $\begin{array}{c}0.264 \\
\mathrm{f} \\
\end{array}$ & $\begin{array}{l}0 \\
\mathrm{j}\end{array}$ \\
\hline \multicolumn{13}{|c|}{$3^{r d}$ cut } \\
\hline Without & $\begin{array}{c}0.381 \\
b\end{array}$ & $\begin{array}{c}0.192 \\
\mathrm{~g}\end{array}$ & $\begin{array}{l}0 \\
\mathrm{~h}\end{array}$ & $\begin{array}{l}0 \\
\mathrm{~h}\end{array}$ & $\begin{array}{l}0 \\
\mathrm{~h}\end{array}$ & $\begin{array}{l}0 \\
\mathrm{~h}\end{array}$ & $\begin{array}{c}0.187 \\
b\end{array}$ & $\begin{array}{c}0.096 \\
\mathrm{e}\end{array}$ & $\begin{array}{l}0 \\
\mathrm{f}\end{array}$ & $\begin{array}{l}0 \\
\mathrm{f}\end{array}$ & $\begin{array}{l}0 \\
\mathrm{f}\end{array}$ & $\begin{array}{l}0 \\
\mathrm{f}\end{array}$ \\
\hline $\begin{array}{l}1 \text { ton/fed } \\
\text { straw }\end{array}$ & $\begin{array}{c}0.397 \\
b\end{array}$ & $\begin{array}{c}0.294 \\
\mathrm{e}\end{array}$ & $\begin{array}{l}0 \\
\mathrm{~h}\end{array}$ & $\begin{array}{l}0 \\
\mathrm{~h}\end{array}$ & $\begin{array}{l}0 \\
\mathrm{~h}\end{array}$ & $\begin{array}{l}0 \\
\mathrm{~h}\end{array}$ & $\begin{array}{c}0.189 \\
\text { b }\end{array}$ & $\begin{array}{c}0.145 \\
\mathrm{~d}\end{array}$ & $\begin{array}{l}0 \\
\mathrm{f}\end{array}$ & $\begin{array}{l}0 \\
\mathrm{f}\end{array}$ & $\begin{array}{l}0 \\
\mathrm{f}\end{array}$ & $\begin{array}{l}0 \\
\mathrm{f}\end{array}$ \\
\hline $\begin{array}{l}2 \text { ton/fed } \\
\text { straw }\end{array}$ & $\begin{array}{c}0.502 \\
\mathrm{a}\end{array}$ & $\begin{array}{c}0.328 \\
\mathrm{~d}\end{array}$ & $\begin{array}{l}0 \\
\mathrm{~h}\end{array}$ & $\begin{array}{l}0 \\
\mathrm{~h}\end{array}$ & $\begin{array}{l}0 \\
\mathrm{~h}\end{array}$ & $\begin{array}{l}0 \\
\mathrm{~h}\end{array}$ & $\begin{array}{c}0.234 \\
\mathrm{a}\end{array}$ & $\begin{array}{c}0.156 \\
\mathrm{~cd}\end{array}$ & $\begin{array}{l}0 \\
\mathrm{f}\end{array}$ & $\begin{array}{l}0 \\
\mathrm{f}\end{array}$ & $\begin{array}{l}0 \\
\mathrm{f}\end{array}$ & $\begin{array}{l}0 \\
\mathrm{f}\end{array}$ \\
\hline Plastic & $\begin{array}{c}0.352 \\
\mathrm{c}\end{array}$ & $\begin{array}{c}0.223 \\
\mathrm{f}\end{array}$ & $\begin{array}{l}0 \\
\mathrm{~h}\end{array}$ & $\begin{array}{l}0 \\
\mathrm{~h}\end{array}$ & $\begin{array}{l}0 \\
\mathrm{~h}\end{array}$ & $\begin{array}{l}0 \\
\mathrm{~h}\end{array}$ & $\begin{array}{c}0.167 \\
\mathrm{c}\end{array}$ & $\begin{array}{c}0.103 \\
\mathrm{e}\end{array}$ & $\begin{array}{l}0 \\
\mathrm{f}\end{array}$ & $\begin{array}{l}0 \\
\mathrm{f}\end{array}$ & $\begin{array}{l}0 \\
\mathrm{f}\end{array}$ & $\begin{array}{l}0 \\
\mathrm{f}\end{array}$ \\
\hline
\end{tabular}

Egypt. J. Agron. 36, No. 1 (2014) 
TABLE 9. Cont.

\begin{tabular}{|c|c|c|c|c|c|c|c|c|c|c|c|c|}
\hline \multicolumn{7}{|c|}{ Dry wt stem } & \multicolumn{6}{|c|}{ Leave / stem ration } \\
\hline \multirow[b]{2}{*}{$\begin{array}{l}\text { Planting } \\
\text { date }\end{array}$} & \multicolumn{3}{|c|}{ 4500ppm } & \multicolumn{3}{|c|}{ 8000ppm } & \multicolumn{3}{|c|}{ 4500ppm } & \multicolumn{3}{|c|}{ 8000ppm } \\
\hline & $1 / 5$ & $15 / 5$ & $1 / 6$ & $1 / 5$ & $15 / 5$ & $1 / 6$ & $1 / 5$ & $15 / 5$ & $1 / 6$ & $1 / 5$ & $15 / 5$ & $1 / 6$ \\
\hline $\begin{array}{l}\text { Soil } \\
\text { mulching }\end{array}$ & \multicolumn{12}{|c|}{$1^{s t} \mathrm{cut}$} \\
\hline Without & $\begin{array}{c}0.270 \\
b-e\end{array}$ & $\begin{array}{c}0.301 \\
\text { b-e }\end{array}$ & $\begin{array}{c}0.385 \\
\mathrm{ab}\end{array}$ & $\begin{array}{c}0.196 \\
\mathrm{e}\end{array}$ & $\begin{array}{c}0.227 \\
\text { de }\end{array}$ & $\begin{array}{c}0.246 \\
\mathrm{de}\end{array}$ & $\begin{array}{c}1.783 \\
\mathrm{ab}\end{array}$ & $\begin{array}{c}1.668 \\
\mathrm{abc}\end{array}$ & $\begin{array}{c}1.249 \\
\text { d-h }\end{array}$ & $\begin{array}{c}1.238 \\
\text { e-h }\end{array}$ & $\begin{array}{c}1.250 \\
\text { d-h }\end{array}$ & $\begin{array}{c}0.920 \\
\text { hi } \\
\end{array}$ \\
\hline $\begin{array}{l}1 \text { ton/fed } \\
\text { traw }\end{array}$ & $\begin{array}{c}0.258 \\
\text { cde }\end{array}$ & $\begin{array}{c}0.261 \\
\text { cde }\end{array}$ & $\begin{array}{c}0.343 \\
\text { a-d }\end{array}$ & $\begin{array}{c}0.222 \\
\text { de }\end{array}$ & $\begin{array}{c}0.214 \\
\mathrm{e}\end{array}$ & $\begin{array}{c}0.255 \\
\text { cde }\end{array}$ & $\begin{array}{c}1.526 \\
b-e\end{array}$ & $\begin{array}{c}1.667 \\
\mathrm{abc}\end{array}$ & $\begin{array}{c}1.527 \\
\text { b-e }\end{array}$ & $\begin{array}{c}1.077 \\
\mathrm{f}-\mathrm{i}\end{array}$ & $\begin{array}{c}1.355 \\
\mathrm{c}-\mathrm{g}\end{array}$ & $\begin{array}{c}0.947 \\
\text { hi }\end{array}$ \\
\hline $\begin{array}{l}2 \text { ton/fed } \\
\text { straw }\end{array}$ & $\begin{array}{c}0.247 \\
\text { de }\end{array}$ & $\begin{array}{c}0.247 \\
\text { de }\end{array}$ & $\begin{array}{c}0.303 \\
\text { b-e }\end{array}$ & $\begin{array}{c}0.211 \\
\mathrm{e}\end{array}$ & $\begin{array}{c}0.225 \\
\text { de }\end{array}$ & $\begin{array}{c}0.199 \\
\mathrm{e}\end{array}$ & $\begin{array}{c}1.885 \\
\mathrm{a}\end{array}$ & $\begin{array}{c}1.556 \\
\text { a-e }\end{array}$ & $\begin{array}{c}1.428 \\
\mathrm{c}-\mathrm{f}\end{array}$ & $\begin{array}{c}1.244 \\
\text { e-h }\end{array}$ & $\begin{array}{c}1.354 \\
\mathrm{c}-\mathrm{g}\end{array}$ & $\begin{array}{c}1.016 \\
\text { ghi }\end{array}$ \\
\hline Plastic & $\begin{array}{c}0.308 \\
\text { b-e }\end{array}$ & $\begin{array}{c}0.377 \\
\mathrm{abc}\end{array}$ & $\begin{array}{c}0.456 \\
\mathrm{a} \\
\end{array}$ & $\begin{array}{c}0.233 \\
\text { de }\end{array}$ & $\begin{array}{c}0.255 \\
\text { cde }\end{array}$ & $\begin{array}{c}0.254 \\
\text { cde }\end{array}$ & $\begin{array}{c}1.603 \\
\text { a-d }\end{array}$ & $\begin{array}{c}1.537 \\
\text { b-e }\end{array}$ & $\begin{array}{c}1.269 \\
\text { d-h }\end{array}$ & $\begin{array}{c}1.254 \\
\text { d-h }\end{array}$ & $\begin{array}{c}1.343 \\
\mathrm{c}-\mathrm{g}\end{array}$ & $\begin{array}{c}0.871 \\
\mathrm{i} \\
\end{array}$ \\
\hline \multicolumn{13}{|c|}{$2^{\text {nd }}$ cut } \\
\hline Without & $\begin{array}{c}0.314 \\
\text { b-e }\end{array}$ & $\begin{array}{c}0.342 \\
\text { a-e }\end{array}$ & $\begin{array}{c}0.291 \\
\text { c-g }\end{array}$ & $\begin{array}{c}0.174 \\
\mathrm{i}\end{array}$ & $\begin{array}{c}0.177 \\
\mathrm{i}\end{array}$ & $\begin{array}{l}0 \\
j\end{array}$ & $\begin{array}{c}1.319 \\
\mathrm{a}\end{array}$ & $\begin{array}{c}1.106 \\
\mathrm{abc}\end{array}$ & $\begin{array}{c}0.878 \\
\text { de }\end{array}$ & $\begin{array}{c}0.855 \\
\text { de }\end{array}$ & $\begin{array}{c}0.984 \\
\mathrm{~cd}\end{array}$ & $\begin{array}{l}0 \\
\mathrm{~g}\end{array}$ \\
\hline $\begin{array}{l}\text { 1ton/fed } \\
\text { straw }\end{array}$ & $\begin{array}{c}0.370 \\
\text { a-d }\end{array}$ & $\begin{array}{c}0.370 \\
\text { a-d }\end{array}$ & $\begin{array}{c}0.325 \\
\text { b-f }\end{array}$ & $\begin{array}{c}0.221 \\
\text { ghi }\end{array}$ & $\begin{array}{c}0.206 \\
\text { hi }\end{array}$ & $\begin{array}{l}0 \\
\mathrm{j}\end{array}$ & $\begin{array}{c}1.219 \\
\mathrm{ab}\end{array}$ & $\begin{array}{c}1.254 \\
\mathrm{a} \\
\end{array}$ & $\begin{array}{c}1.025 \\
\text { bcd }\end{array}$ & $\begin{array}{c}0.940 \\
\mathrm{~cd}\end{array}$ & $\begin{array}{c}1.023 \\
\text { bcd }\end{array}$ & $\begin{array}{l}0 \\
\mathrm{~g}\end{array}$ \\
\hline $\begin{array}{l}2 \text { ton/fed } \\
\text { straw }\end{array}$ & $\begin{array}{c}0.416 \\
\mathrm{a}\end{array}$ & $\begin{array}{c}0.421 \\
\mathrm{a} \\
\end{array}$ & $\begin{array}{c}0.285 \\
\text { d-h }\end{array}$ & $\begin{array}{c}0.305 \\
\text { b-g }\end{array}$ & $\begin{array}{c}0.245 \\
\mathrm{f}-\mathrm{i} \\
\end{array}$ & $\begin{array}{l}0 \\
\mathrm{j}\end{array}$ & $\begin{array}{c}1.308 \\
\mathrm{a}\end{array}$ & $\begin{array}{c}1.179 \\
\mathrm{ab}\end{array}$ & $\begin{array}{c}1.260 \\
\mathrm{a} \\
\end{array}$ & $\begin{array}{c}0.645 \\
\mathrm{f}\end{array}$ & $\begin{array}{c}1.015 \\
\text { bcd }\end{array}$ & $\begin{array}{l}0 \\
\mathrm{~g}\end{array}$ \\
\hline Plastic & $\begin{array}{c}0.390 \\
\mathrm{ab}\end{array}$ & $\begin{array}{c}0.374 \\
\mathrm{abc}\end{array}$ & $\begin{array}{c}0.349 \\
\text { a-e } \\
\end{array}$ & $\begin{array}{c}0.304 \\
\text { b-g }\end{array}$ & $\begin{array}{c}0.275 \\
\text { e-h }\end{array}$ & $\begin{array}{l}0 \\
\mathrm{j}\end{array}$ & $\begin{array}{c}1.319 \\
\mathrm{a}\end{array}$ & $\begin{array}{c}1.211 \\
\mathrm{ab}\end{array}$ & $\begin{array}{c}0.731 \\
\text { ef }\end{array}$ & $\begin{array}{c}0.715 \\
\text { ef }\end{array}$ & $\begin{array}{c}0.965 \\
\mathrm{~cd}\end{array}$ & $\begin{array}{l}0 \\
\mathrm{~g}\end{array}$ \\
\hline & & & & & & ${ }^{r d}$ cut & & & & & & \\
\hline Without & $\begin{array}{c}0.194 \\
\mathrm{~b} \\
\end{array}$ & $\begin{array}{c}0.096 \\
\mathrm{f} \\
\end{array}$ & $\begin{array}{l}0 \\
\mathrm{~g}\end{array}$ & $\begin{array}{l}0 \\
\mathrm{~g}\end{array}$ & $\begin{array}{l}0 \\
\mathrm{~g}\end{array}$ & $\begin{array}{l}0 \\
\mathrm{~g}\end{array}$ & $\begin{array}{c}0.971 \\
\mathrm{a}\end{array}$ & $\begin{array}{c}1.007 \\
\mathrm{a} \\
\end{array}$ & $\begin{array}{l}0 \\
\mathrm{c}\end{array}$ & $\begin{array}{l}0 \\
\mathrm{c}\end{array}$ & $\begin{array}{l}0 \\
\mathrm{c}\end{array}$ & $\begin{array}{l}0 \\
\mathrm{c}\end{array}$ \\
\hline $\begin{array}{l}\text { 1ton/fed } \\
\text { straw }\end{array}$ & $\begin{array}{c}0.193 \\
\mathrm{~b}\end{array}$ & $\begin{array}{c}0.149 \\
\mathrm{~d}\end{array}$ & $\begin{array}{l}0 \\
\mathrm{~g}\end{array}$ & $\begin{array}{l}0 \\
\mathrm{~g}\end{array}$ & $\begin{array}{l}0 \\
\mathrm{~g}\end{array}$ & $\begin{array}{l}0 \\
\mathrm{~g}\end{array}$ & $\begin{array}{c}0.986 \\
\mathrm{a}\end{array}$ & $\begin{array}{c}0.980 \\
\mathrm{a} \\
\end{array}$ & $\begin{array}{l}0 \\
\mathrm{c}\end{array}$ & $\begin{array}{l}0 \\
\mathrm{c}\end{array}$ & $\begin{array}{l}0 \\
\mathrm{c}\end{array}$ & $\begin{array}{l}0 \\
\mathrm{c}\end{array}$ \\
\hline $\begin{array}{l}2 \text { ton/fed } \\
\text { straw }\end{array}$ & $\begin{array}{c}0.248 \\
\mathrm{a} \\
\end{array}$ & $\begin{array}{c}0.172 \\
\mathrm{c}\end{array}$ & $\begin{array}{l}0 \\
\mathrm{~g} \\
\end{array}$ & $\begin{array}{l}0 \\
\mathrm{~g}\end{array}$ & $\begin{array}{l}0 \\
\mathrm{~g}\end{array}$ & $\begin{array}{l}0 \\
\mathrm{~g}\end{array}$ & $\begin{array}{c}0.958 \\
\mathrm{a} \\
\end{array}$ & $\begin{array}{c}0.908 \\
\mathrm{ab} \\
\end{array}$ & $\begin{array}{l}0 \\
\mathrm{c}\end{array}$ & $\begin{array}{l}0 \\
\mathrm{c} \\
\end{array}$ & $\begin{array}{l}0 \\
\mathrm{c}\end{array}$ & $\begin{array}{l}0 \\
\mathrm{c} \\
\end{array}$ \\
\hline Plastic & $\begin{array}{c}0.185 \\
\text { bc }\end{array}$ & $\begin{array}{c}0.121 \\
\mathrm{e}\end{array}$ & $\begin{array}{l}0 \\
\mathrm{~g}\end{array}$ & $\begin{array}{l}0 \\
\mathrm{~g}\end{array}$ & $\begin{array}{l}0 \\
\mathrm{~g}\end{array}$ & $\begin{array}{l}0 \\
\mathrm{~g}\end{array}$ & $\begin{array}{c}0.904 \\
\mathrm{ab}\end{array}$ & $\begin{array}{c}0.854 \\
\mathrm{~b}\end{array}$ & $\begin{array}{l}0 \\
\mathrm{c}\end{array}$ & $\begin{array}{l}0 \\
\mathrm{c}\end{array}$ & $\begin{array}{l}0 \\
\mathrm{c}\end{array}$ & $\begin{array}{l}0 \\
\mathrm{c}\end{array}$ \\
\hline
\end{tabular}

\section{References}

Abd El-Rahman, S.M., El-Shouny, K.A., Ashoub, M.A., Abd El-Gawad, M.A. and Abd El- Maaboud, M.Sh. (2005) Effect of salinity and nitrogen bio-fertilization on some Sudan grass (Sorghum sudananse L. Moench) varieties at Ras Sudr. Arab. Univ. J. Agric. Sci. Ain Shams Univ. Cairo, 13 (3), 755-769

Blummel, M., Zerbini, E., Reddy, B.V., Hash, C.T., Bidinger, F. and Khan, A.A. (2003) Improving the production and utilization of sorghum and pearl millet as livestock feed: progress towards dual-purpose genotypes. Field Crops Res. 84,143158. 
Chapman, H.D. and Pratt, P.F. (1961) "Methods and Analysis for Soils, Plants, and Waters". Univ. Calif. Div. Agr. Sci., Berkeley, 309.

Ferraris, R. (1973) Pearl millet (Pennisetum typhoides). Commonwealth Bureau of Pastures and Field Crops (no. 1/1973). Slough: C.A.B.(c.f. FAO Plant Prot Bull,(1983). 31 (4), 136-158.

Hancock, D.W. and Greg Durham, R. (2010) Late Planting Date Influences the Yield and Distribution of Pearl Millet Forage. Forage and Grazinglands, 8 (1). (c.f. ARPN J. of Sci. and Tech (2013). 3 (4): 340-344.)

Hussein, M.M., Abdel-Kader, A.A., Kady, K.A., Youssef, R.A. and Alva, A.K. (2010) Sorghum response to foliar application of phosphorus and potassium with saline water irrigation. J. Crop Improvement, 24 (4), 324-336.

Jackson, M.L. (1958) "Soil Chemical Analysis". Prentice-Hall, Englewood Cliffs, NJ, USA.

Kobayashi, Y., Yato, S., Fujikaya, T., Nakamura, T., Mihara, M. and Komamura, M. (2010) Effects of stubble mulching on plant growth of pearl millet and soil moisture condition. IJEAD., 1 (2), 44-48.

Krishnamurthy, L., Serraj, R., Rai, K.N., Hash, C.T. and Dakheel, A.J. (2007) Identification of pearl millet [Pennisetum glaucum (L.) $R$. Br.] lines tolerant to soil salinity. Euphytica, 158 (1-2), 179-188.

Leila Radhouane (2013) Agronomic and physiological responses of pearl millet ecotype (Pennisetumglaucum (L.) R. Br.) to saline irrigation. Emir. J. Food Agric. 25 (2), 109116.

Maas, A.L., Hanna, W.W. and Mullinix, B.G. (2007) Planting date and row spacing affects grain yield and height of pearl millet Tifgrain 102 in the Southeastern coastal plain of the United States. J. SAT Agric Research, 5 (1), 1-4.

Radhouane, L. (2008) Autochthonous pearl millet ecotype (Pennisetum glaucum LR BR.) respons to different sowing dates in Tunisia. Sjemenarstvo, 25 (2), 123-138.

Siti Aishah, H.A., Saber, R., Halim, R.A. and Zaharah, A.R. (2011). Yield responses of forage sorghums to salinity and irrigation frequency. African J. Biotechnology, $\mathbf{1 0}$ (20), 4114-4120.

Soler, C.M.T., N.Maman, X. Zhang, S.C. Mason, and Hoogenboom, G. (2008) Determining optimum planting dates for pearl millet for two contrasting environments using a modelling approach. The J. Agric Sci, 146 (04), 445-459.

Steel, R.C. and Torrie, J.H. (1960) Principles and Procedures of Statistics, McGrawHill. 481.pp (c.f. Biology of reproduction, (1980). 22 (5), 1062-1067).

Uwahm, D.F. and Iwo, G.A. (2011) Effectiveness of organic mulch on the productivity of maize (Zea mays L.) and weed growth. J. Anim. Plant Sci. 21(3), 525-530. 
Wailare, M.A. (2009) Responses of pearl millet (Pennisetum flaucum) to varying sowing date and variety at Bagauda, Kano State, Nigeria. Journal of Agriculture, Forestry and the Social Sciences, 7 (2). (c.f. ARPN J. of Sci. and Tech, (2013). 3 (4), 340-344).

Waller, R.A. and Duncan, D.B. (1969) A bays rule for the segmmetric multiple comparison problem. J. Amer. Stat. Assoc., 64 (328), 1484-1503.

Wang, H., Zhang, L., Dawes, W.R. and Liu, C. (2001) Improving water use efficiency of irrigated crops in the north China plain-measurements and modeling. Agric. Water Manage, 48 (2),151-167.

Yakubu H., Ngala, A.L. and Dugje, I.Y. (2010) Screening of millet (Pennisetum glaucum $L$.) varieties for salt tolerance in semi-arid soil of northern Nigeria. World $J$. Agric. Sci. 6 (4), 374-380.

Zeinolabedin Jouyban (2012) The Effects of Salt stress on plant growth. Tech. J. Engin. \& App. Sci. 2 (1), 7-10.

(Received 21/5/2014; accepted 10/8/2014) 


\section{تأثير تغطية سطح التربة ومواعيد الزراعة على نمو وإنتاجية الاخن تحت الظروفت الملحية التربة}

$$
\begin{aligned}
& \text { محمد شكرى رياض ، مها متولى عباس حماده ، محسن شحاته عبد المعبود" و }
\end{aligned}
$$

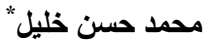

$$
\begin{aligned}
& \text { كلية الزراعة - جامعله عين شمس و "قسم الانتاج النباتى- مركز بحوث }
\end{aligned}
$$

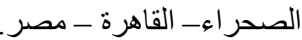

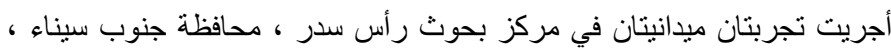

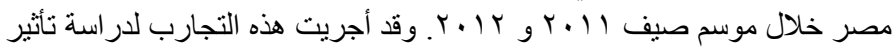

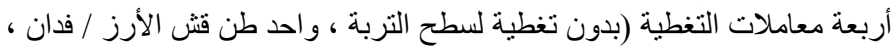

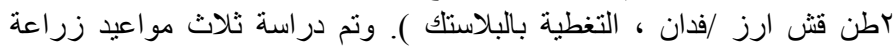

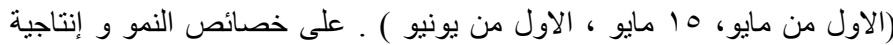

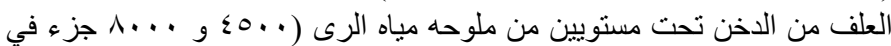

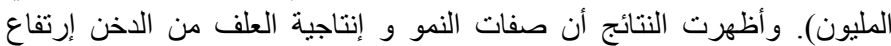

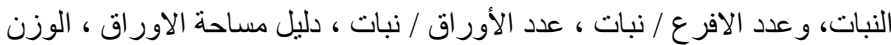

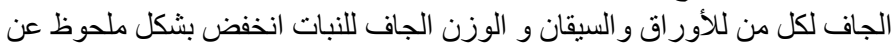

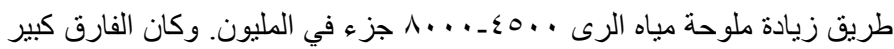

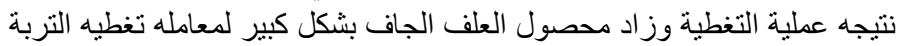

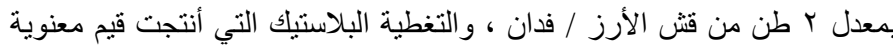

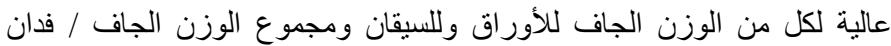

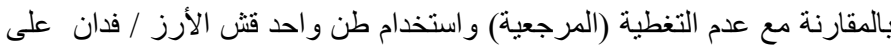

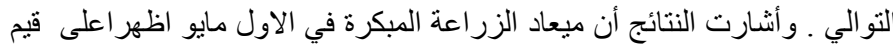
معنوية لمعظم صفات النمو ومحصول العلف الجاف الكلى. 